Tafani Roberto ${ }^{1}$, Chiesa Gastón², Caminati Raul $^{3}$, Gaspio Nuri ${ }^{4}$

${ }^{1}$ Ph.D Social Sciences; Profesor Titular Economía. FCE.UNRC.

2Lic. En Ciencias Políticas. Docente. FCE. UNRC ${ }^{3}$ Lic en Administración de Empresas. Docente. FCE. UNRC

${ }^{4}$ Doctora en Bioquímica

Trabajo recibido: 25 de agosto 2016. Aprobado: 20 de octubre

\section{BIENESTAR HUMANO Y SERVICIOS DE SALUD}

\author{
BEM-ESTAR HUMANO E SERVIÇOS DE SAÚ- \\ $D E$ \\ HUMAN WELFARE AND HEALTH SERVICES
}

Resumen

Se describen los cambios en Argentina en la expectativa de vida 2000-2010 y la mortalidad infantil 2000-2014. Se indaga la asociación de esas variables con el producto geográfico bruto por habitante, la educación de la mujer, la razón de médicos por habitantes y la tasa de utilización de la consulta en establecimientos públicos. Los resultados indican que la mayor asociación con la esperanza de vida al nacer se da con el PGB y la educación de la madre. En ambos casos es débil la asociación con la mortalidad infantil. El número de médicos y las tasas de uso de consultas relacionan muy débilmente con la expectativa de vida al nacer. Los médicos no se asocian con la mortalidad infantil y la tasa de uso de consultas lo hace muy débilmente. La importancia de la mortalidad infantil perinatal y congénita sugiere un futuro más complejo, especializado y costoso que el actual.

Palabras clave: Bienestar, servicios de salud, esperanza de vida, Argentina

\section{Abstract}

Argentina changes in life expectancy and infant mortality 2000-2010 2000-2014 are described. The association of these variables with the gross state product per capita, education of women, the ratio of doctors per capita and the rate of use of consultation in public establishments is investigated. The results indicate that the greatest association with life expectancy at birth is given with the PGB and education of the mother. In both cases the association with infant mortality is weak. The number of 
doctors and usage rates of consultations relate very weakly with life expectancy at birth. The importance of perinatal and infant mortality congenital suggests that the current more complex, specialized and expensive future.

Key words: Welfare, health services, life expectancy, Argentina

\section{Resumo}

Descrevem-se as mudanças na Argentina acerca da expectativa de vida 2000-2010 e mortalidade infantil 2000-2014. Pesquisa-se a associação destas variáveis com o Produto Geográfico Bruto per capita, a educação das mulheres, a proporção de médicos por habitantes e a taxa de utilização de consultas em estabelecimentos públicos. Os resultados indicam que a maior associação com a expectativa de vida no nascimento é dado com o PGB e a educação da mãe. Em ambos os casos é fraca a associação com a mortalidade infantil. O número de médicos e as taxas de uso de consultas relacionam-se muito fracamente com a expectativa de vida ao nascer. Os médicos não são associados com a mortalidade infantil e a taxa de utilização de consultas o faz muito fracamente. A importância de mortalidade infantil perinatal e congênita sugere um futuro mais complexo, especializado e dispendioso do que o atual.

Palavras-chave: Bem-estar, serviços de saúde, expectativa de vida, Argentina.

\section{Antecedentes}

En el último siglo el progreso en salud fue tan importante como el aumento de la riqueza. La esperanza de vida en los países ricos aumentó 30 años y sigue aumentando cada década. Según la Organización Mundial de la Salud, la esperanza de vida aumentó cinco años desde el año 2000. Así, la esperanza de vida global para los niños nacidos en 2015 es de 71,4 años, 73,8 para las mujeres y 69,1 años para los hombres (1). Las mejoras en salud crean brechas, como el progreso material crea diferencias en los estándares de vida. Cuando surgen nuevos conocimientos y tecnologías, hay grupos que son los primeros en beneficiarse mientras otros demoran más tiempo para sumarse y mejorar. En el medio queda un período donde las características son progreso y desigualdad. Esa desigualdad se encuentra asociada a los territorios donde se nace. En los países de altos ingresos, la expectativa de vida es de 80 años o más, mientras que en otras regiones es menor a 60 años.

Las claves del progreso son el conocimiento, las invenciones y las nuevas maneras de hacer las cosas. Deaton (2015) (2), en su libro sobre Salud, riqueza y desigualdad refiere al bienestar como todas las cosas que hacen que la vida para una persona sea buena. Esto incluye no sólo el bienestar material tales como el ingreso y la riqueza sino también el bienestar físico y psicológico representado por la salud y la educación además de la capacidad de participar en la sociedad civil a través de la democracia y el imperio de la ley. En décadas recientes ha habido un gran progreso, más niños asisten a la escuela con regularidad y el número de personas alfabetizadas es mayor, también hay más libertad política que hace medio siglo (Deaton, 2015) (2). Entre los años 1970 y 2010 el mundo sufrió transformaciones notables. Curiosamente ese período ha sido caracterizado como neoliberal porque recompuso las condiciones de rentabilidad para el capital global y la concentración del ingreso en manos de un grupo cada vez menor en el mundo. Al mismo tiempo la esperanza de vida creció de 59 a 70 años, la alfabetización se incrementó del $60 \%$ al $83 \%$ de la población, la matriculación escolar combinada, primaria y secundaria, pasó del 55\% al 70\%, y el ingreso per cápita promedio se elevó de 5.100 a 10.600 dólares (3). Toda una paradoja que parece contradictoria en sí misma. La mayor desigualdad 
económica y mayor concentración del poder que trajo aparejada la consolidación del capitalismo mundial fue acompañada por un período de gran progreso social caracterizado por vidas más largas de personas cada vez más alfabetizadas y educadas en un mundo con ingresos promedios duplicados.

Hasta los críticos del capitalismo que piensan como superarlo deben partir de este reconocimiento. Así (Mason Paul 2016) escribe "El capitalismo es también un organismo que aprende: se adapta constantemente y no solo de un modo gradualista. En momentos que constituyen importantes puntos de inflexión, muta y se transforma en respuesta al peligro, creando a partir de entonces pautas y estructuras nuevas que la generación anterior apenas si lograría reconocer. Y su instinto de supervivencia más básico es el consistente en impulsar el cambio tecnológico. Si además de las tecnologías de la información, tenemos en cuenta también ámbitos como la producción alimentaria, el control de la natalidad o la salud global, veremos que en los últimos veinticinco años han sido probablemente los de mayor aumento de la capacidad humana en toda la historia". (4)

Argentina no fue ajena a estas transformaciones. Con un Producto Bruto Interno per cápita de 12.510 dólares, para el año 2014, el Banco Mundial califica al país como de ingreso alto (5) entre los no pertenecientes al OCDE. La esperanza de vida al nacer calculada durante el año 2010 es de 75 años, promedio para ambos sexos (6). La tasa neta de escolaridad secundaria de la mujer es del $89 \%$, siendo el índice mujeres/varones de 1,10 (7); Según PNUD, 2015 (8), por su alto índice de desarrollo humano $(0,836)$ para el año 2014 Argentina ocupa el puesto 39 entre los 198 países del mundo, hallándose primero en el listado de América Latina, acompañado por Chile que se halla dos lugares más abajo. Dentro de los indicadores, la tasa de mortalidad de la niñez es clave para la expectativa de vida. La tasa de mortalidad de menores de 5 años, se ubica en Argentina debajo de la mediana entre los países del mundo. Si bien no se alcanzaron las metas del milenio de "disminuir en $2 / 3$ partes la tasa de mortalidad de menores de cinco años", el mundo tampoco lo logró. A decir verdad, mientras el mundo en su totalidad redujo el indicador en un 53\% Argentina acompaño esa mejora al pasar de $28 \%$ a $12,2 \%$ nacidos vivos, con una disminución del 54\% (9). En América Latina y El Caribe las cifras fueron aún mejores logrando la región una reducción de la tasa de mortalidad de menores de 5 años del 69\%. Se pasó así, de 54\%o a 17\% nacidos vivos. Pese a los logros de Argentina, el país se sitúa aún detrás de Chile y Uruguay que se hallan en 8,2\% y 11,2\%o respectivamente. En los tres casos, las principales causas de muerte de menores de 5 años son perinatales y congénitas (10). Estas cifras muestran el enorme progreso social que ha acompañado la acumulación de capital en el mundo mediante un sistema que solo permite el acceso a los bienes y servicios a través del poder de compra que da el dinero. La sociedad productora de plusvalía, de relaciones sociales de clases antagónicas cada vez más desiguales y responsable de una devastación medioambiental sin precedentes, lo hizo.

\section{Objetivos, Metodo, Fuentes y Resultados}

Este trabajo tiene por objetivo describir los cambios en Argentina en la esperanza de vida al nacer y la mortalidad infantil por un lado, y la asociación de esas variables con el producto regional per cápita, la educación de la mujer, la razón médicos por habitantes y la tasa de uso de consulta médica por el otro. Mediante la exploración de varios indicadores de salud se analizan las desigualdades en los 24 distritos políticos de Argentina. Su alcance es descriptivo y correlacional y el diseño retrospectivo longitudinal. La técnica utilizada es el análisis de factores. Los datos surgen del Banco Mundial (5), Unicef (7), 
Ministerio de Salud $(6,11)$, y Ministerio de Hacienda y Finanzas Públicas de la República Argentina (12,13).

\section{Esperanza de vida al nacer}

El gráfico de países en función de la esperanza de vida al nacer y el ingreso per cápita, muestra la relevancia inicial del ingreso para aumentar el bienestar. Luego esa variable parece perder relevancia.

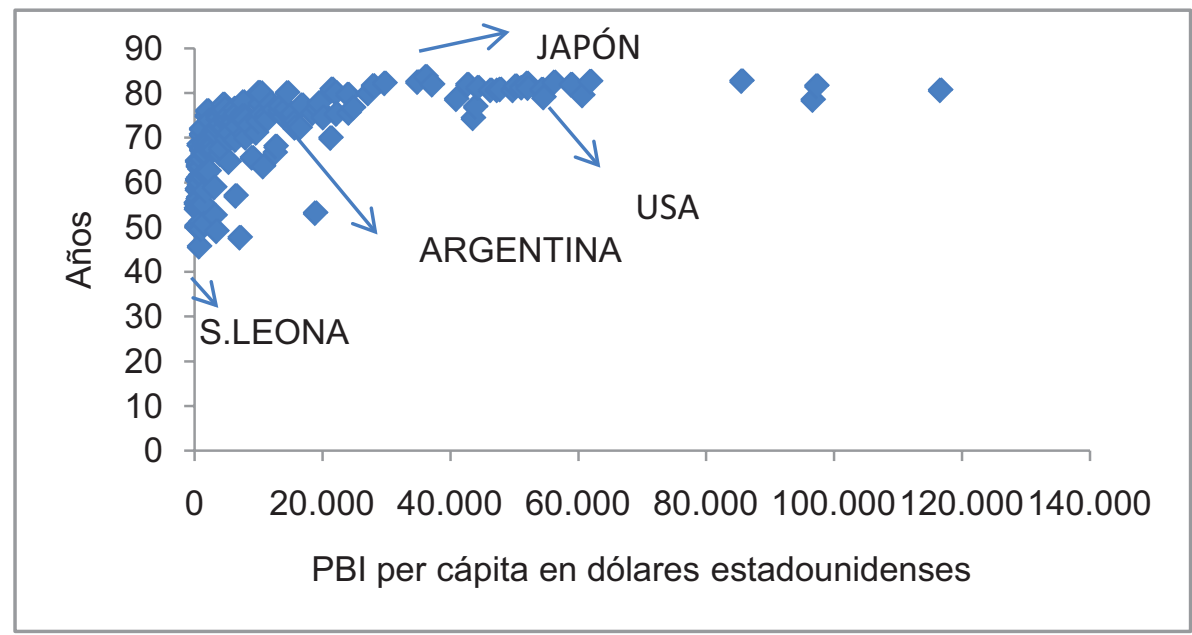

Gráfico No 1. Esperanza de vida al nacer y PBI per cápita, 177 Países, año 2014

Elaboración propia. Fuente de datos: Banco Mundial 2016, (5)

Argentina que comparativamente tiene aún años de esperanza de vida por ganar, está a la derecha del punto de inflexión de la curva. Ese punto, se interpreta como el cambio en el perfil epidemiológico de un país al pasar de las enfermedades infecciosas a las crónico degenerativas. Es el momento en que "la muerte envejece", porque las muertes de los niños son poco comunes y la mayoría corresponde a ancianos.

En el gráfico $\mathrm{N}^{\circ} 2$ se observa la diferencia entre provincias Argentinas en la esperanza de vida al nacer para ambos sexos medida en dos períodos diferentes. Desde el año 2000 al 2010 la expectativa de vida aumentó en 3 años promedio. Estos cambios han sido muy grandes en Provincias que tienen un ingreso por habitante muy distinto. Jujuy y Tierra del Fuego por ejemplo, ganaron 7 años cada una. Salta y Neuquén seis años cada una. En La Rioja, Catamarca, Chubut y San Luis la cifra fue de 5 años. Como se verá más adelante, la sistemática comparación entre ingreso y expectativa de vida por Provincias da una correlación que evidencia que el PGB determina casi el 50\% de la esperanza de vida al nacer $(\mathrm{R} 2=49 \%)$ lo que no explica sin embargo, como Provincias con niveles de riqueza tan disímiles como Jujuy y Tierra del fuego logran similares progresos en el indicador.

${ }^{6}$ Esperanza de vida al nacer, Tasa de mortalidad de menores de 5años, Nivel de educación de la madre, Producto bruto geográfico, Servicios de salud públicos y privados con y sin internación, médicos, camas, tasas de uso de consulta médica ambulatoria en servicios oficiales, ausencia de cobertura de obra social, servicios de agua potable y cloacas. 


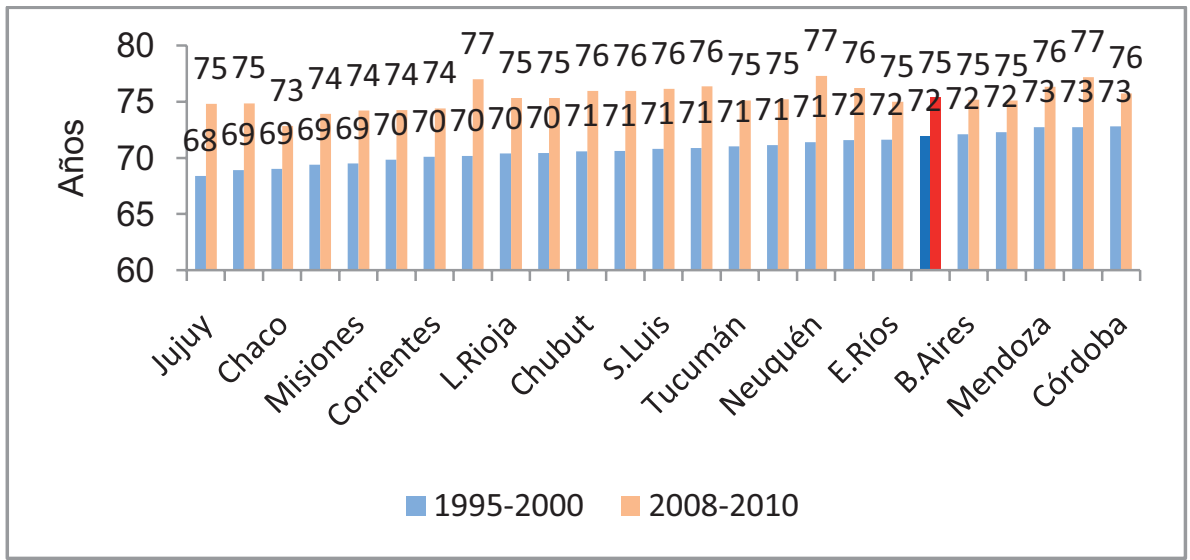

Gráfico No 2. Esperanza de vida al nacer, Argentina 2000 y 2010

Elaboración propia. Fuente de datos. Ministerio de Salud. Argentina OPS 2003 y 2015. (6).

En el año 2000 la diferencia entre el valor menor (Jujuy) y el mayor (Córdoba) era de 5 años o del 7\%. Diez años después, la diferencia entre el valor mayor (Neuquén) y el valor menor (Chaco) es de 4 años o sea del $5 \%$ entre ambas provincias. Esto muestra que la desigualdad se achica.

\section{Mortalidad de la niñez y adulta}

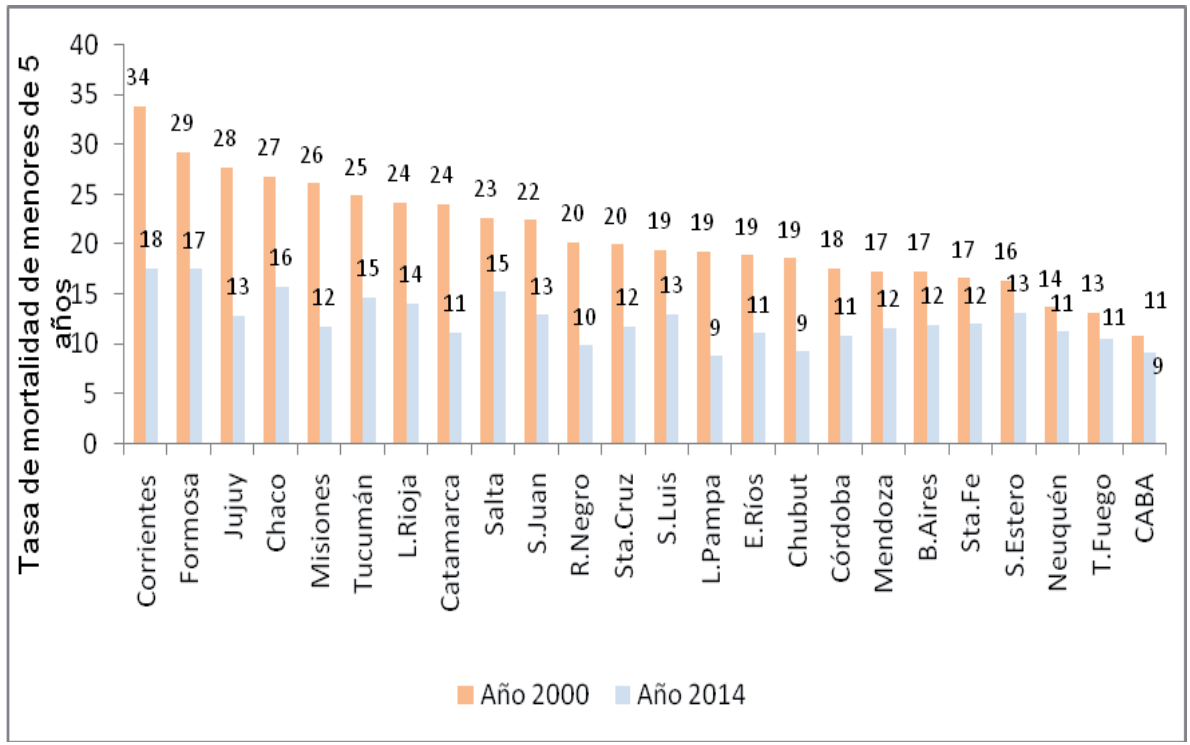

Gráfico No 3. Tasa de Mortalidad de Menores de cinco años. Argentina 2000 y 2014 Elaboración propia. Fuentes de datos: Fuente de datos. Ministerio de Salud.

Estadísticas Vitales, Años 2001 y 2015. (11)

Las más altas tasas para ambos periodos se mantienen en Formosa, Chaco, Corrientes y Salta, es decir las provincias del NEA y una del NOA. Jujuy, Misiones, Catamarca, Santiago del Estero, San Juan y San Luis bajan apreciablemente. Córdoba que también cae 
se ubica por debajo del promedio país que es de $12,2 \%^{\circ}$. La Pampa presenta el valor más bajo. En los gráficos $\mathrm{N}^{\circ} 4$ y N5 se observa el Índice de Gini que mide la inequidad entre provincias en los años analizados.

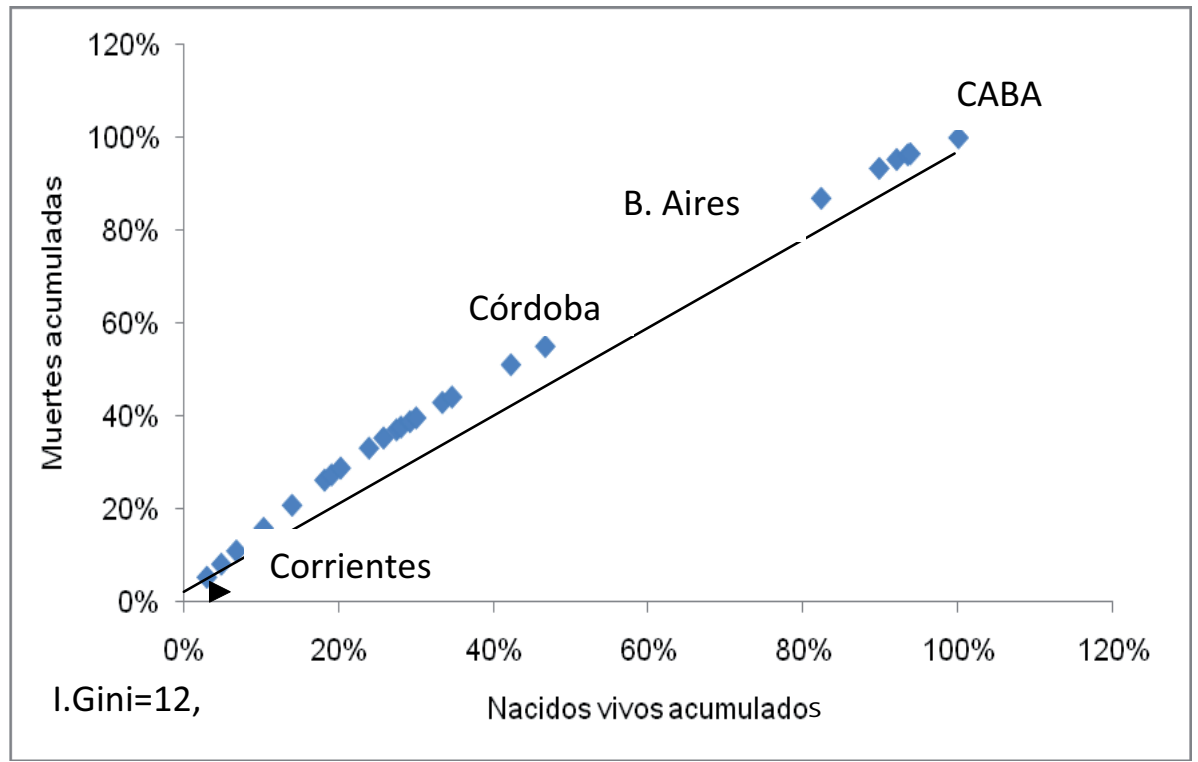

Gráfico Nº4: Índice de Gini. Mortalidad de la niñez. Argentina 2000

Elaboración propia. Fuentes de datos: Fuente de datos. Ministerio de Salud.

Estadísticas Vitales, Años 2001 y 2015. (11)

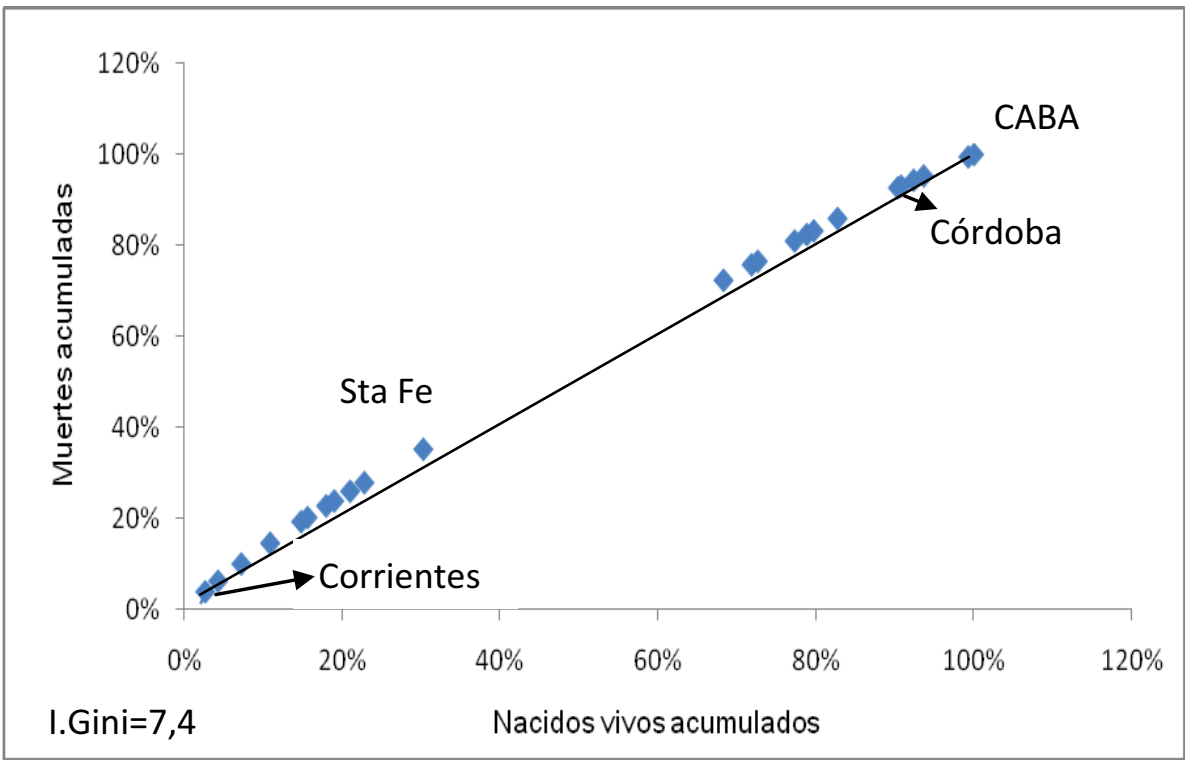

Gráfico No5: Índice de Gini. Mortalidad de la niñez. Argentina 2014

Elaboración propia. Fuentes de datos: Fuente de datos. Ministerio de Salud.

Estadísticas Vitales, Años 2001 y 2015. (11)

El indicador ha disminuido en el periodo en estudio, de 12,6\% a 7,4\% o sea cayó en un $41 \%$. Eso se observa visualmente en el aplanamiento de la curva de puntos. Nuevamente se verifica la caída de la desigualdad. La diferencia entre la provincia con mayor tasa de mortalidad y la menor mide la muerte por inequidad social. Esto corresponde a Corrientes y CABA y ha pasado de 3,1 a 1,9 Dicho de otra forma, durante el año 2000 de cada 4 
niños que morían 3 de esas muertes se debían a la inequidad social, mientras que catorce años después de cada 3 muertes menos de 2 corresponden a la diferencia social entre uno y otro lugar.

Otra manera de observar la evolución de la situación de salud puede obtenerse mediante la relación entre la muerte de menores de 5 años y la de mayores de 60 años en la mortalidad total. En el año 2000 la misma fue del 4,9\% para los niños y 73,7\% en los mayores de 60 años. Una década y media después en el año 2014 las cifras cambian marcadamente y bajan al 2,9 \% las muertes de los niños en la mortalidad total, mientras crecen al 78,4\% en los adultos. Esto significa que hubo una reducción del $40 \%$ en la participación de la mortalidad total de los niños menores de cinco años y un crecimiento del $6 \%$ en los mayores de sesenta.

Para evaluar mejor el cambio impactante de esos indicadores, hay que recordar que, Deaton (2) p.132, ha encontrado que este valor es de $35 \%$ para niños y $27 \%$ para mayores de 60 años en los países pobres y $0,9 \%$ para niños, contra $83,8 \%$ para mayores de 60 años en los países ricos. Ubicado en ese contexto, Argentina se encuentra próxima a los valores de los países que hoy muestran los mejores valores de mortalidad y expectativa de vida en el mundo. Puesto en magnitudes absolutas durante el año 2000 en Argentina murieron 13.533 menores de 5 años, siendo la tasa de mortalidad de la niñez de 19,3* \%o nacidos vivos. Catorce años después murieron 9.480 menores de 5 años $(12,2 * \%$ ) y la tasa de variación negativa fue del (-37\%). (11)

El gráfico $\mathrm{N}^{\mathrm{o}} 6$ muestra como la caída de la participación de la mortalidad de la niñez en la total se da a partir del año 2003, y sigue disminuyendo hasta 2014, excepto en el año 2008, que hace un pequeño ascenso. Toda esta baja se da inicialmente con tasas de crecimiento en el producto bruto interno cercanas al $9 \%$ anual. En el 2008 la tasa de variación del producto comienza a decrecer, alcanzando un mínimo en 2009 que coincide con la disminución del porcentaje de muertes de mayores de 60 años. Luego en los años finales las tasas negativas de crecimiento no impiden que la mortalidad siga progresando en la dirección deseada (menos participación de niños y más de adultos mayores en la mortalidad total)

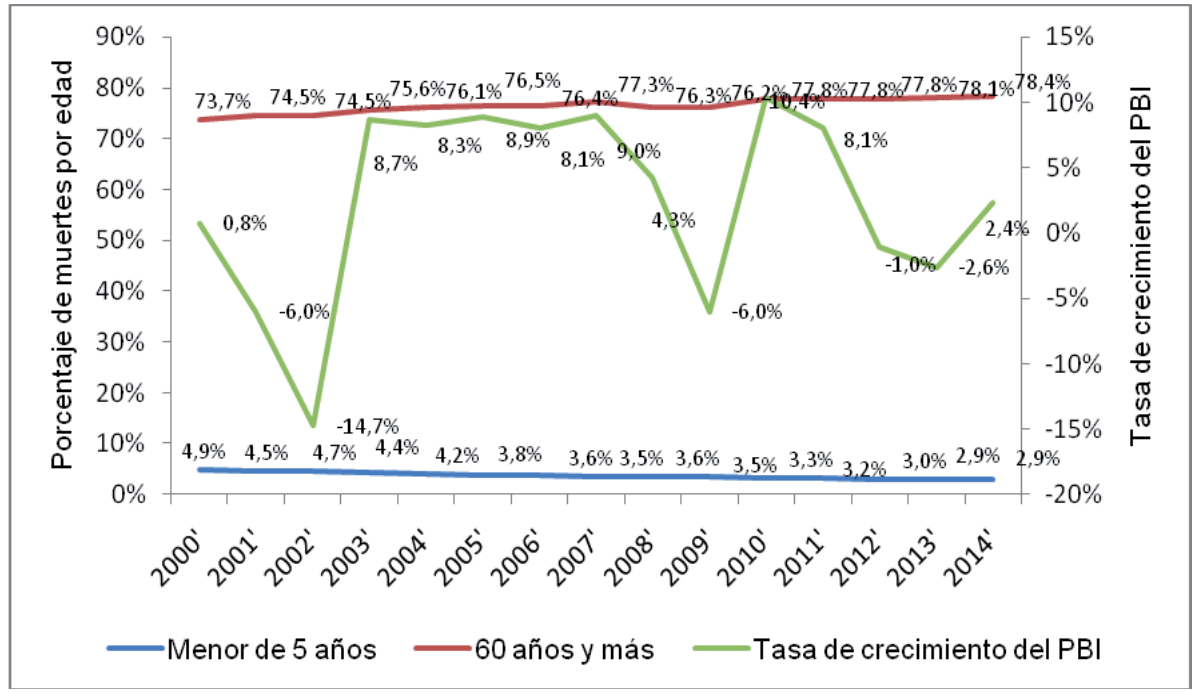

Gráfico $\mathrm{N}^{\mathrm{o}}$ 6: Porcentaje de mortalidad de menores de 5 años, mayores de 60 años y tasa de crecimiento del PBI

Elaboración propia. Fuente de datos. Ministerio de Salud. Estadísticas Vitales, 2001,2002,2003,2004,2005,2006,2007,2008,200 9,2010,2011,2012,2013,2014,2015. (11) Indec 2016(15).

La implicancia parece ser que las bajas y la estabilización de las muertes se dan en 
contextos de crecimiento económico muy distintos. Así el PBI decreció -6\% en 2009,$1 \%$ en 2012 y $-2,6 \%$ en 2014 , mientras que el porcentaje de muertes de menores de 5 años sigue bajando en el total y crece el porcentaje de muertes de mayores de 60 . La participación de la mortalidad de la niñez en la total parece entonces no responder a las tasas de crecimiento de la riqueza.

La conclusión hasta aquí es que la salud pública medida por la expectativa de vida al nacer ha hecho un enorme progreso en los últimos 15 años, que ese progreso se reparte con valores muy altos entre Provincias de muy desigual ingreso por habitante, que la desigualdad entre Provincias ha disminuido y que el envejecimiento de la muerte medida por la participación de dos grupos etarios (menores de 5 años y mayores de 60) no parecen vincularse con la dinámica macroeconómica del país. Queda obviamente un camino a recorrer pero es evidente que la salud pública ha mostrado signos incontrastables de progreso en lo que va del siglo 21 .

Para entender mejor como ha ocurrido ese proceso se desagrega la mortalidad de los menores de 5 años. Como se mencionara antes (en relación a Uruguay y Chile), se observa que alrededor del $44 \%$ corresponde a causas perinatales.

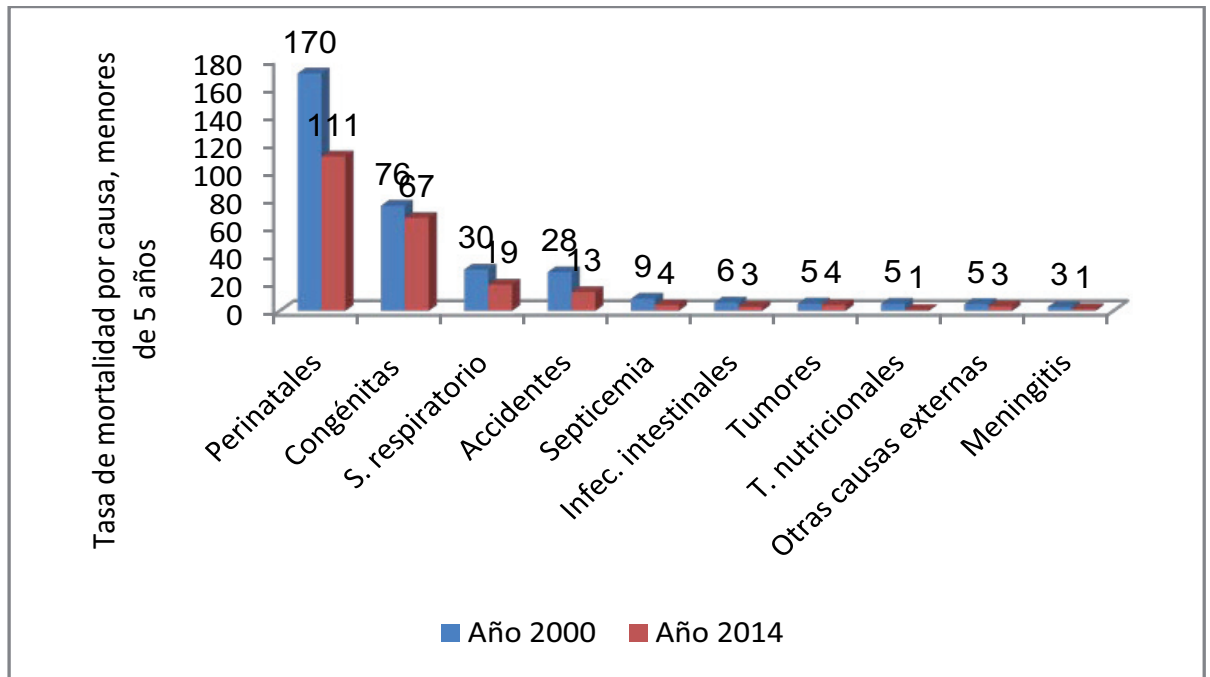

Gráfico $\mathrm{N}^{\mathrm{o}} 7$ : Tasa de mortalidad por causa de menores de 5 años* 100.000 nacidos vivos . Argentina 2000 y 2014

Elaboración propia. Fuente de datos. Ministerio de Salud. Estadísticas Vitales. Años 2001 y 2015. (11)

En el gráfico $\mathrm{N}^{\circ} 8$ se observa la variación de la tasa de mortalidad por causa.

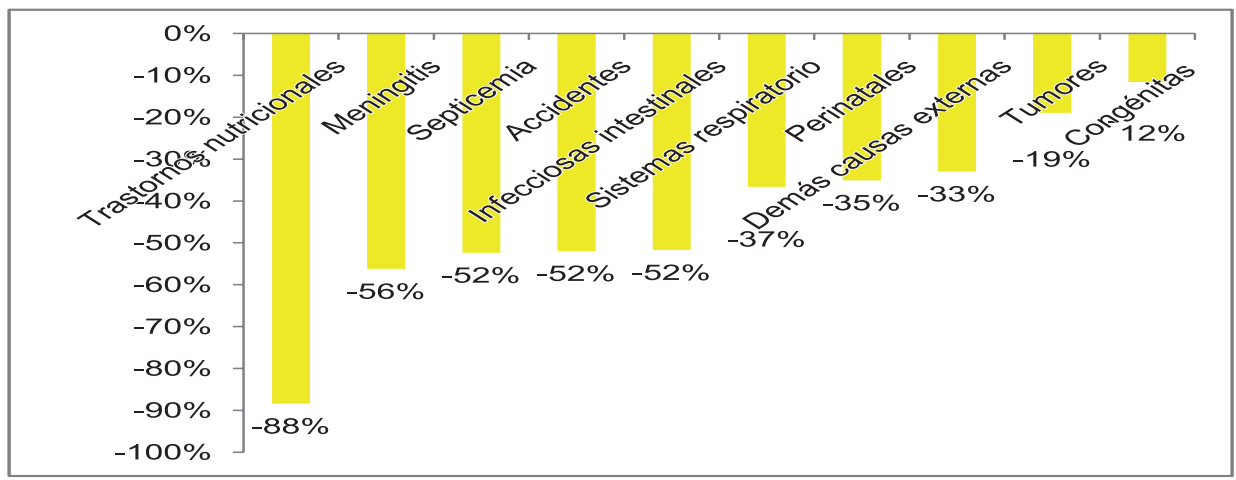

Gráfico No 8: Tasa de variación de la mortalidad por causa año 2000 y 2014

Elaboración propia. Fuentes de datos: Fuente de datos. Ministerio de Salud.

Estadísticas Vitales. Años 2001 y 2015. (11) 
Para la mortalidad de menores de 5 años, entre los años 2000 y 2014 las bajas más importantes (superiores al $50 \%$ ) se observan en trastornos nutricionales, meningitis, septicemia, e infecciones intestinales. En proporciones más bajas han disminuido todas las demás siendo la que menos varió la muerte por enfermedades congénitas.

En el año 2014 el 28\% de muertes perinatales (entre la vigésima octava semana de gestación y el séptimo día de nacimiento) se debió a trastornos relacionados con la duración de la gestación y el crecimiento fetal (11). La muerte perinatal es el resultado final de la interacción de una serie de factores presentes en el proceso de gestación. Existe en ella un componente social relacionado con el acceso económico o educativo de la madre así como la oportunidad y eficiencia de los servicios de salud (16). Según un estudio llevado a cabo en siete centros colaboradores de la OMS, entre los que se hallaba Argentina el parto pre término espontáneo y los trastornos hipertensivos fueron los casos obstétricos más comunes asociados a las defunciones perinatales $(28,7 \%$ y $23,6 \%$, respectivamente). La prematuridad fue la causa principal de las defunciones neonatales precoces (62\%) (17). Paris E. (2011) (18), reportó que el 98\% de las muertes perinatales ocurren en los países pobres, pero los países ricos no están libres de esta tragedia en la que un 30\% de los casos siguen sin tener una causa conocida. Cada año se producen en el mundo más muertes durante la gestación que por enfermedades como el sida y paludismo juntas.

Pasando a las muertes de los mayores de 60 años, en lo que va del siglo, hubo un aumento de muertes por enfermedades del aparato genitourinario, en las enfermedades del aparato respiratorio y digestivo mientras que en circulatorio y tumores hay un descenso. Los gráficos siguientes describen la situación.

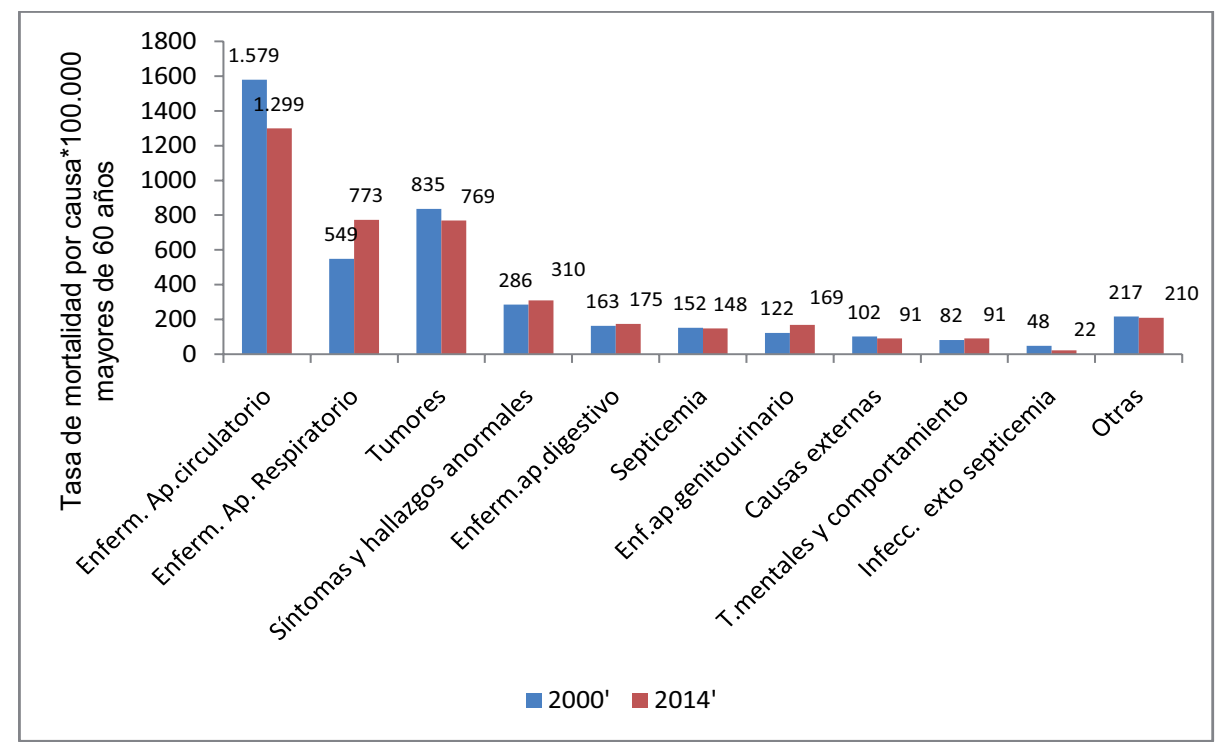

Gráfico $\mathrm{N}^{\circ}$ 9: Tasa de mortalidad por causa del adulto de 60 años y más.

Argentina años 2000 y2014

Elaboración propia. Fuentes de datos Fuente de datos. Ministerio de Salud.

Estadísticas Vitales. Años 2001 y 2015. (11)

En el gráfico $\mathrm{N}^{0} 10$ se observa la estructura proporcional de muertes por tumores, la cual no tiene casi variación. Las prevalentes en 2014 son: tráquea, bronquio y pulmón (15,7\%), seguido del cáncer de colon (11,2\%). Si sumamos a esos dos grupos el cáncer de mama

${ }^{8}$ La tasa de mortalidad de Menores de cinco años es la probabilidad que un recién nacido cada 1.000, muera antes de cumplir cinco años de edad, si está sujeto a las tasas de mortalidad específicas por edad del año especificado. (14)

${ }^{9}$ Tasa de mortalidad por causa de menores de 5 años = Número de niños de 0 a 4 años muertos por una enfermedad determinada* $100.000 /$ número de niños de 0 a 4 años a mitad del año en estudio. 
$(8,6 \%)$ de próstata $(7,7 \%)$, páncreas $(7 \%)$ estómago y útero $(5 \%)$ se tiene casi el $58 \%$ del total contra el $56 \%$ en el año 2000. La distribución de las patologías permanece casi invariable en su estructura proporcional

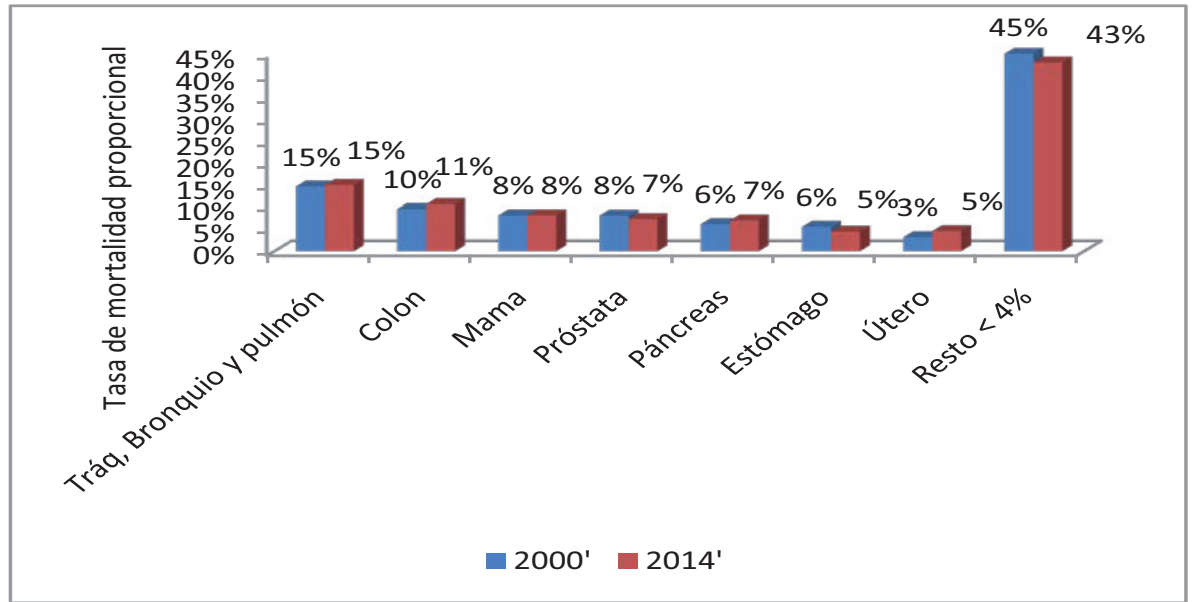

Gráfico $\mathrm{N}^{\mathrm{o}}$ 10: Tasa de mortalidad proporcional por tumores

Elaboración propia. Fuentes de datos Fuente de datos. Ministerio de Salud.

Estadísticas Vitales. Años 2001 y 2015. (11)

Hasta aquí hemos visto los cambios en expectativa de vida y la mortalidad. Esos cambios parecen estar débilmente relacionados con los cambios en el crecimiento económico. ¿Qué es entonces lo que puede explicar los hechos documentados? Una posible explicación es que esto tenga que ver con la riqueza provincial, la educación de la mujer o la atención médica que han tenido cambios importantes. Seguidamente exploramos las asociaciones respectivas.

\section{Producto Geográfico Bruto per cápita}

Se observan en el gráfico $\mathrm{N}^{\circ} 11$ las diferencias entre los PGB per cápitas correspondientes a 1999 y 2014, las ocho provincias más pobres pertenecen al NOA y NEA, mientras que las cuatro más ricas, luego de CABA, son patagónicas.

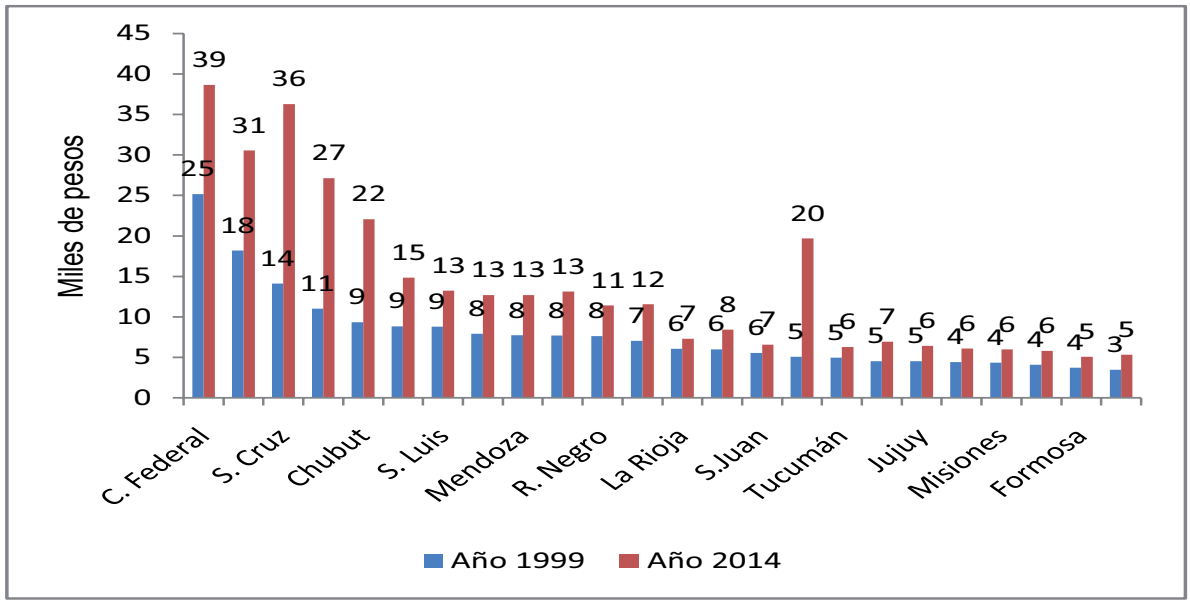

Gráfico N 11 : Producto geográfico bruto Argentina 1999 y 2014

Elaboración propia. Fuentes de datos. INDEC. Sistema de Indicadores de Desarrollo Provincial. Años 2000 y 2015. (12) 
La cuestión que interesa es como se relaciona ese producto con los indicadores de salud. El gráfico siguiente incorpora justamente la expectativa de vida al nacer y los resultados indican que el Producto bruto geográfico per cápita determina casi un 50\% de la esperanza de vida al nacer, el resto se debería ajustar por otro indicador. Si bien entonces hay una asociación entre las dos variables hay que retomar aquí lo planteado anteriormente, Tierra del Fuego tiene una riqueza por habitante varias veces superior a la de Jujuy pero ambas tuvieron incrementos similares de siete años en los cambios en la expectativa de vida al nacer. Lo mismo pasa con Salta y Neuquén.

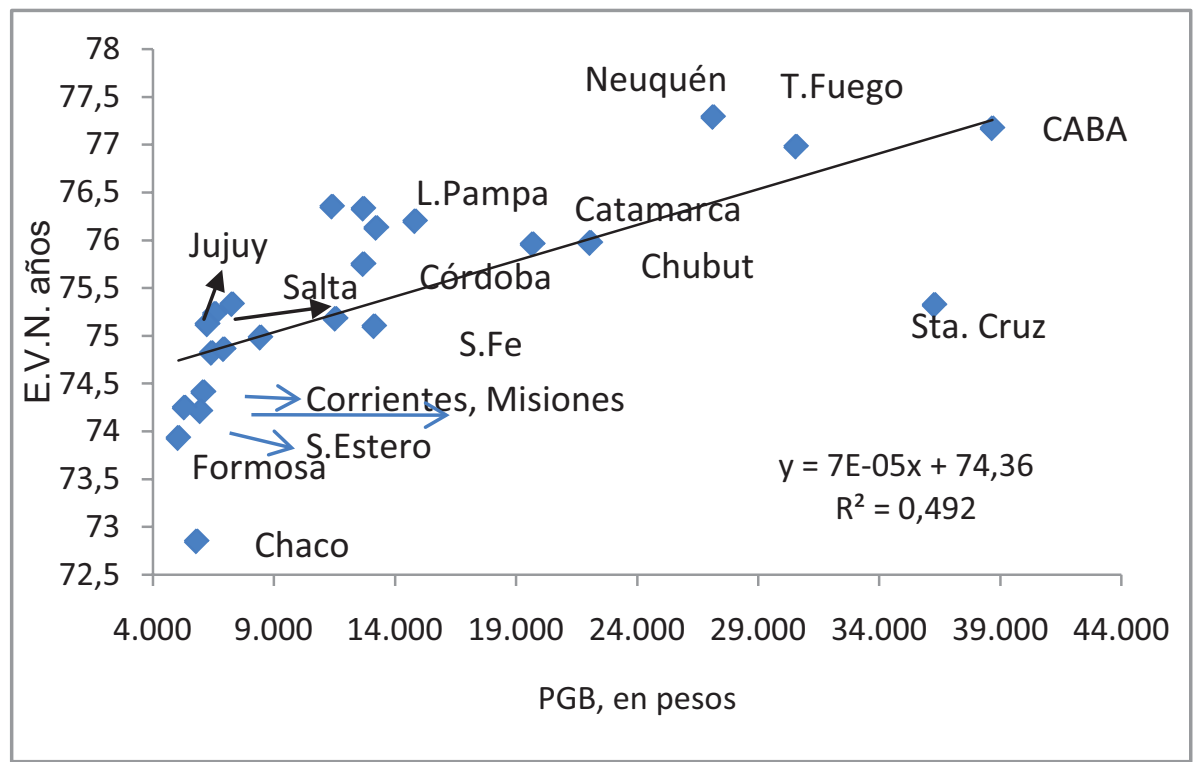

Gráfico N¹2: Relación entre PGB por cápita y expectativa de vida al nacer

Elaboración propia. Fuentes de datos. INDEC. Sistema de Indicadores de Desarrollo Provincial, 2015 (12). . Ministerio de Salud. Estadísticas Vitales. Año 2015. (11)

Mientras la riqueza por habitante de Salta es muy cercana a la de Jujuy, Neuquén se encuentra como Tierra del Fuego varias veces por encima de aquella. Sin embargo como se mostrara antes, los progresos en expectativa de vida fueron en ambos casos de seis años en cada una de esas Provincias. Los ejemplos siguen. Formosa una de las provincias con menor producto geográfico bruto tiene como Santa Cruz y Chubut que son varias veces más ricas, un incremento en la expectativa de vida de cinco años. Lo mismo pasa entre Chaco y la Ciudad Autónoma de Buenos Aires cada una en un extremo opuesto de la distribución del producto pero ambas con un incremento en la expectativa de vida de cuatro años. Claramente no alcanza con el argumento basado en el producto geográfico bruto por habitante. Hay mucha distancia económica entre los pares de Provincias citadas y sin embargo el progreso en expectativa de vida es similar entre ellas. Si esta limitación de la variable económica se manifiesta en la imposibilidad de acompañar una explicación robusta sobre los progresos en expectativa de vida en zonas de distinto nivel de riqueza, la cuestión es aún más notable cuando se relaciona el producto con la mortalidad infantil. Esto, porque cuando al producto geográfico bruto se lo relaciona con la tasa de mortalidad infantil, el impacto es bastante menor. La riqueza determina un $35 \%$ de la segunda variable y el resto tendría que ser explicado por otras variables. Pasemos entonces a otras variables 


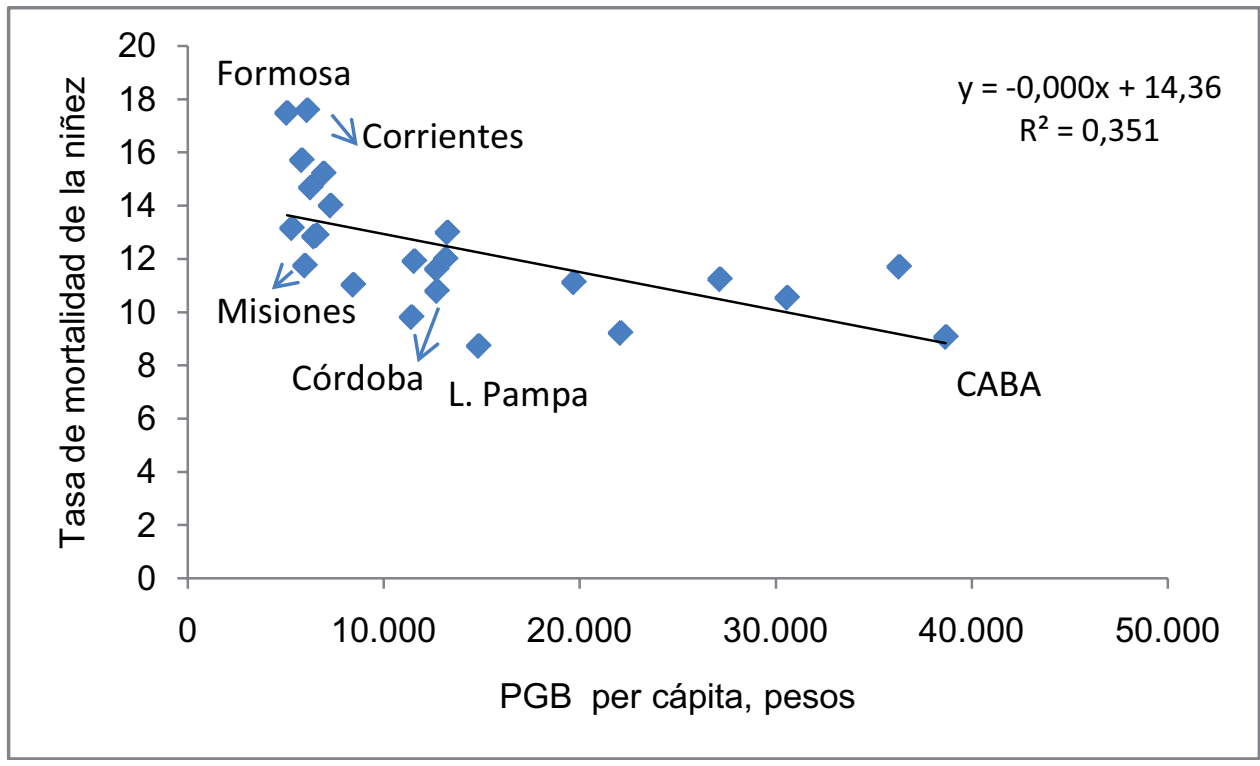

Gráfico $\mathrm{N}^{\mathrm{o}}$ 13: Tasa de mortalidad de la niñez/PGB per cápita

Elaboración propia. Fuentes de datos. INDEC. Sistema de Indicadores de Desarrollo Provincial, 2015 (12). Ministerio de Salud. Argentina OPS 2015. (6)

\section{Educación de la mujer}

Datos de la Encuesta Permanente de Hogares del tercer trimestre de 2003 y tercer trimestre de 2014 (INDEC) nos muestran que entre 2003 y 2014, disminuyó el porcentaje de mujeres de 16 años o más sin instrucción o primario incompleto de $11,8 \%$ a $7,3 \%$, primario completo de $42,4 \%$ a $38,7 \%$, mientras que aumentó el porcentaje de mujeres con secundario completo de $32,4 \%$ a $39,6 \%$ y terciario o universitario completo de $13,3 \%$ a $17,1 \%$. Es decir en estos 15 años hubo avances importantes en la educación de la mujer. El gráfico $\mathrm{N}^{\circ} 14$ visualiza que si bien ha disminuido la proporción de hijos de madres sin instrucción o con primario incompleto y también la de madres con primario completo, esta última categoría sigue siendo más alta que la de madre con secundario completo, que aumentó moderadamente. Ello probablemente se deba a que las madres con mayor nivel de instrucción suelen tener menos hijos. También hubo un leve aumento en las madres con terciario o universitario completo. De los niños nacidos durante el año 2000, el 7\% (51.333) eran por lo menos el sexto hijo, de ellos el 32\% de las madres no había completado primaria y el 6\% había completado el secundario. Durante 2014 nacieron un $3 \%$ (24541) de niños que eran el sexto hijo o más, de ellos el $21 \%$ de las madres no completó la primaria y el $11 \%$ de las madres completó la secundaria. (11) (Estadísticas Vitales). 


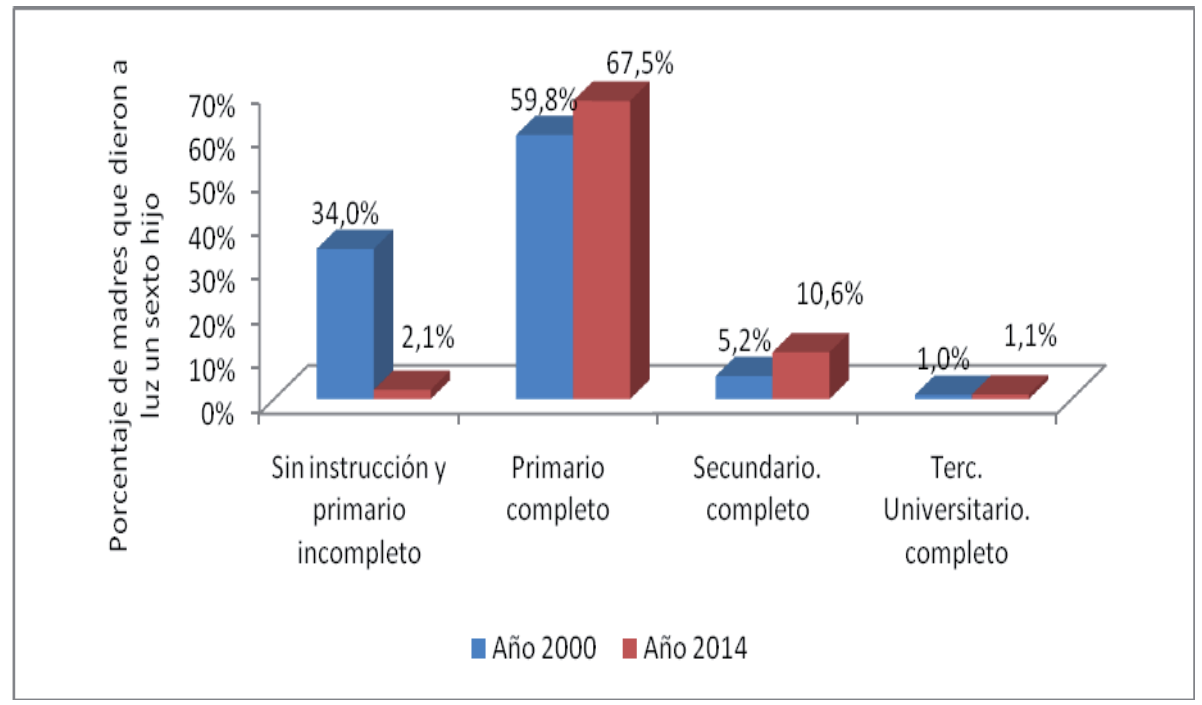

Gráfico N N 14: Nivel de educación de las madres de nacidos vivos 2007 y 2014

Elaboración propia. Fuentes de datos Fuente de datos. Ministerio de Salud.

Estadísticas Vitales. Años 2001 y 2015. (11)

Como se relaciona la mayor educación de la mujer con la esperanza de vida al nacer es lo que describe el grafico que sigue.

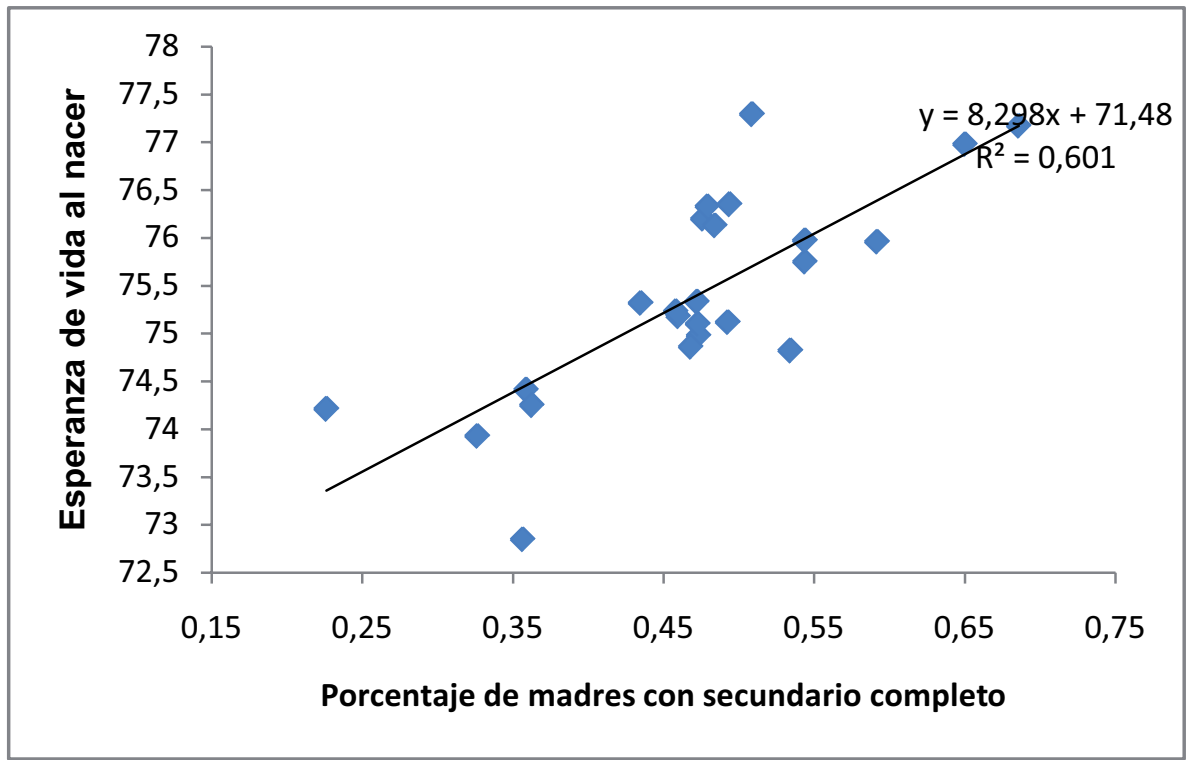

Gráfico $\mathrm{N}^{\mathrm{o}}$ 15: Relación entre madres con secundario completo y esperanza de vida al nacer

Elaboración propia. Fuentes de datos Fuente de datos. Ministerio de Salud.

Estadísticas Vitales. Años 2015. (11). Argentina OPS 2015 (6)

La asociación es significativa. El gráfico $\mathrm{N}^{0} 15$ pone en evidencia, la fuerte correlación entre la educación secundaria de la madre y la esperanza de vida al nacer, el primer indicador determina en un $60 \%$ al segundo. La asociación con la mortalidad infantil sin embargo es mucho menos marcada. 


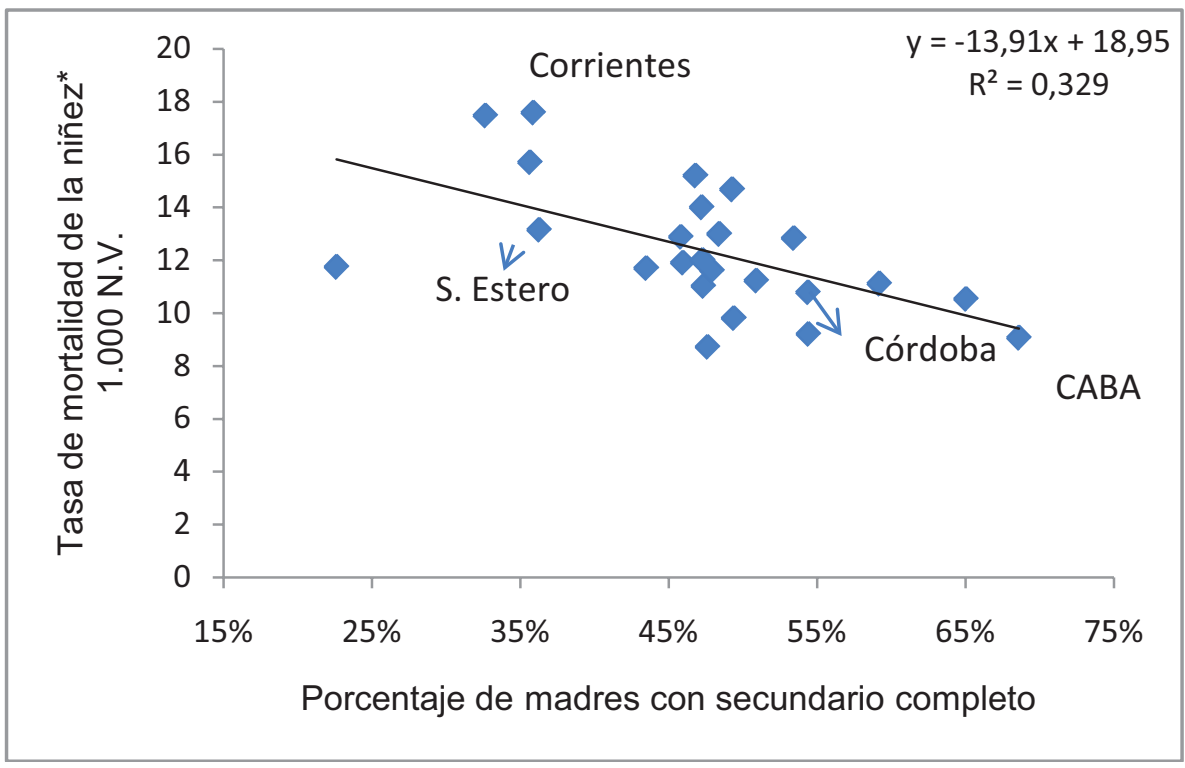

Gráfico $\mathrm{N}^{\mathrm{o}} 16$ : Relación entre la tasa de mortalidad de menores de 5 años y educación de la madre

Elaboración propia. Fuentes de datos Fuente de datos. Ministerio de Salud.

Estadísticas Vitales. Años 2015. (11)

Se observa en el gráfico $\mathrm{N}^{\circ} 16$ que la relación entre el nivel de educación secundario completo de la madre determina en un $33 \%$ la tasa de mortalidad de la niñez. Por otro lado en los diez años en estudio, la educación secundaria de las madres creció en un $48 \%$ mientras que la tasa de mortalidad de la niñez decreció en (-38\%). Posiblemente lo que esté ocurriendo es que al ser cada vez mayor la presencia de las muertes por causas congénitas, ni la riqueza provincial ni la educación de la mujer se asocien a esa distribución de frecuencia de muertes que ocurren por causas más complejas e inevitables.

\section{Médicos y tasas de uso de consulta médica}

Argentina cuenta con alrededor de 4 médicos cada 1.000 habitantes. En el gráfico $\mathrm{N}^{\circ}$ 17 se puede observar la distribución por regiones para los dos periodos en estudio, las proporciones son más bajas en NOA y NEA y se observa la migración hacia Patagonia, que ha duplicado la proporción de médicos en 10 años. 


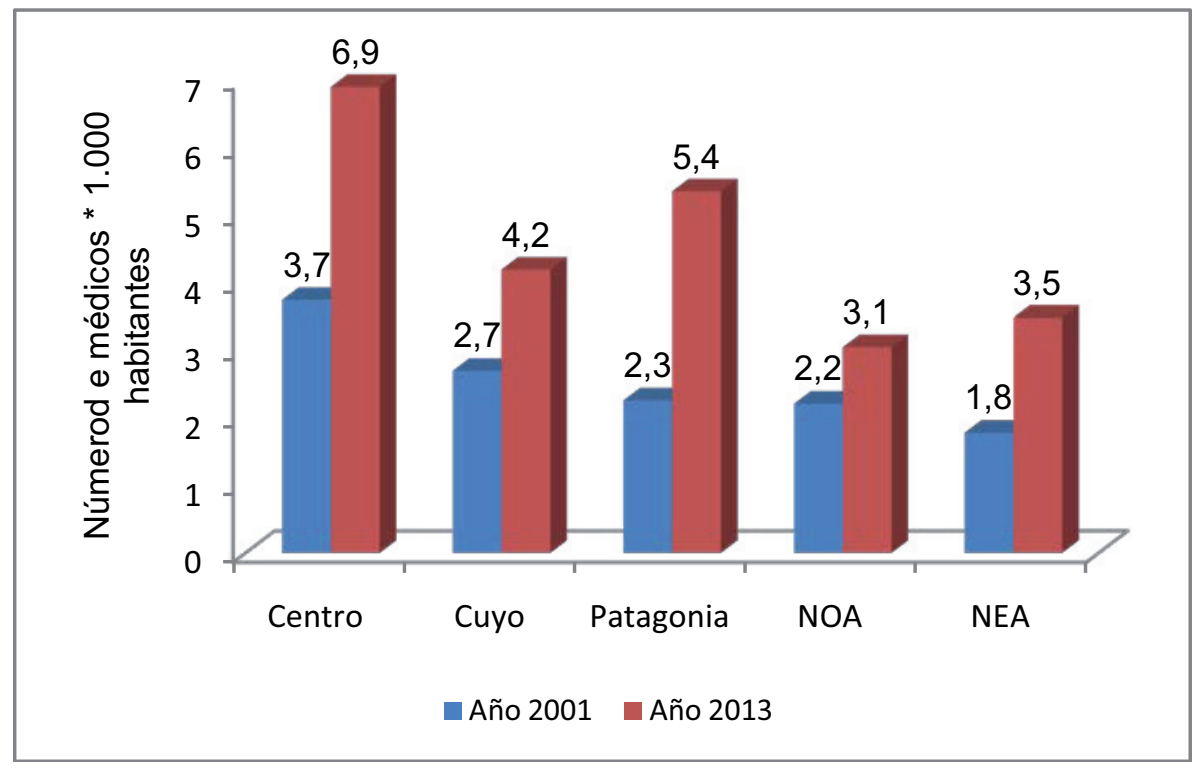

Gráfico N 17: Razón de médicos por 1000 habitantes en las regiones de Argentina 2000 y 2013

Elaboración propia. Fuentes de datos: Ministerio de Salud. Observatorio Federal de Recursos Humanos en Salud 2015 (19).

En el gráfico $\mathrm{N}^{\circ} 18$ se observa la distribución de médicos por provincia, el alto número en CABA se debe a que se contabilizan en esa columna los médicos con matrícula nacional, que les es necesaria para trabajar en los hospitales nacionales. Tierra del Fuego, Río Negro y Neuquén son las provincias con mayor aumento en el número de médicos en 15 años. Los médicos parecen migrar hacia las provincias patagónicas que como se vio tienen un mayor PGB per cápita. Siguen en dos palabras la ruta del dinero.

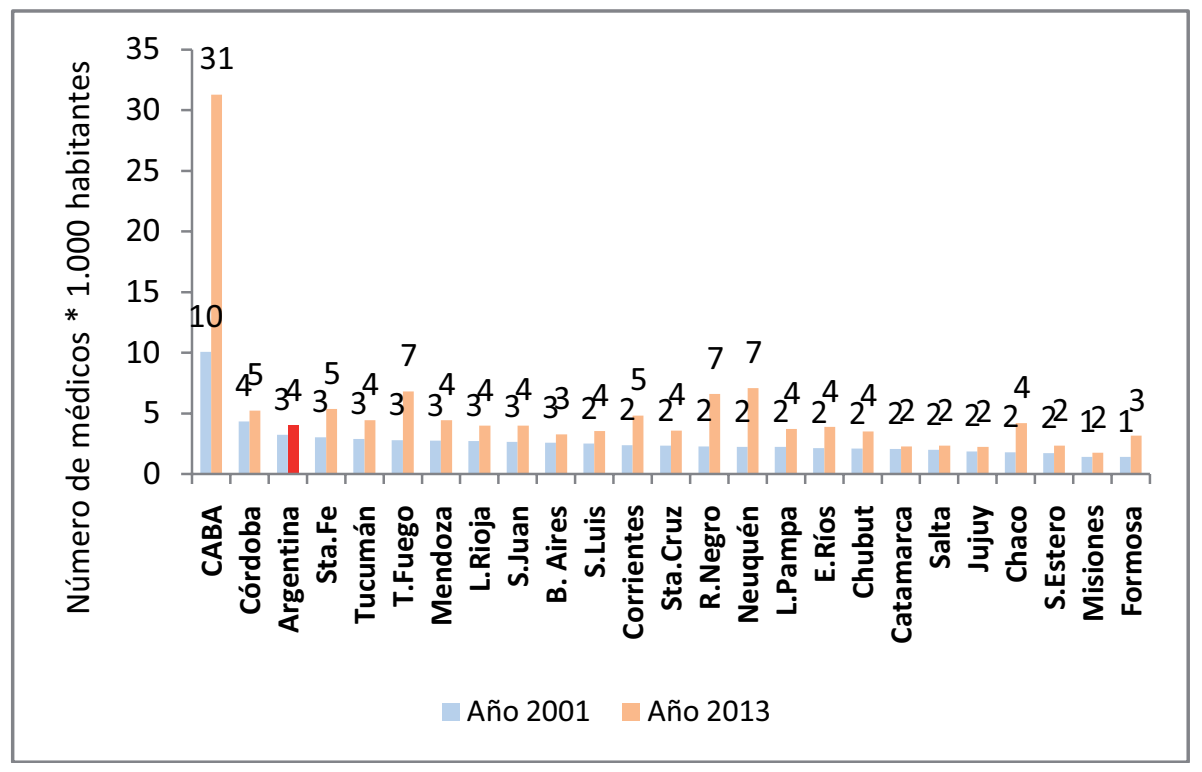

Gráfico Nº18: Médicos cada 1.000 habitantes por provincias.Argentina 2000 y 2014 Elaboración propia. Fuentes de datos Ministerio de Salud. Observatorio Federal de Recursos Humanos en Salud 2015 (19). 
Como se hizo antes con las otras variables en el gráfico que sigue se vincula la razón de médicos por habitantes con la expectativa de vida.

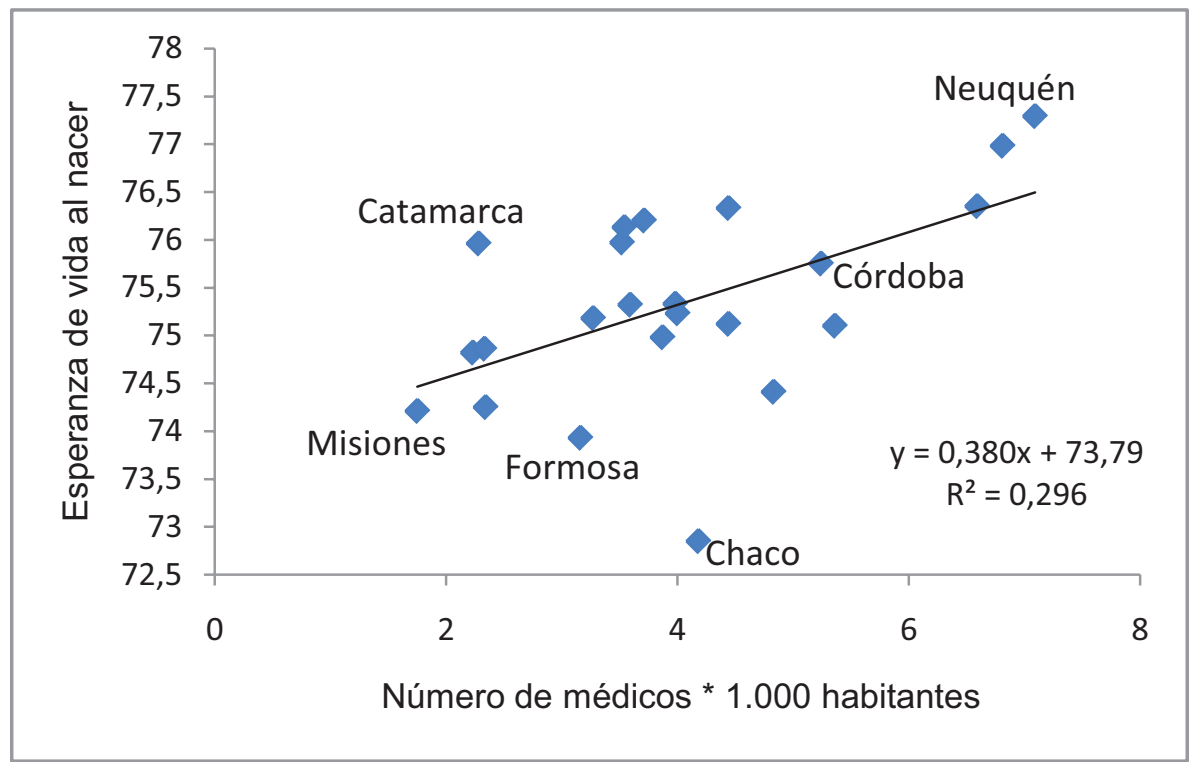

Gráfico No 19: Relación entre médicos cada 10.000 habitantes y esperanza de vida al nacer Elaboración propia. Fuentes de datos Ministerio de Salud. Argentina OPS 2015 (6) Observatorio Federal de Recursos Humanos en Salud 2015 (19).

El gráfico $\mathrm{N}^{\circ} 19$ muestra que la relación entre los médicos por 1.000 habitantes y la esperanza de vida al nacer determinan en un $29,6 \%$ de la esperanza de vida al nacer. Es la menos relevante de todas las asociaciones vistas hasta aquí. Por otro lado la relación entre el número de médicos y la tasa de mortalidad de la niñez, presenta una correlación muy pequeña y estadísticamente no significativa $(4,8 \%)$, como venimos aseverando en otros trabajos. Así las cosas, lo que parece ocurrir es que las variables analizadas tienen en todos los casos mayor asociación cuando refieren a la expectativa de vida que a la mortalidad infantil, y el cuadro que surge es uno donde lo central es la mayor educación de la mujer, la riqueza provincial que no explica enormes disparidades de riqueza pero similar avance en años esperados de vida y la poca y nula relevancia que tiene la razón médicos por habitantes en relación a la expectativa de vida y la mortalidad infantil respectivamente. Finalmente resta explorar la tasa de utilización de los servicios.

\section{Tasa de uso de consulta ambulatoria}

En el gráfico $\mathrm{n}^{0} 20$ se observa que las tasas de uso de consulta ambulatoria en servicios oficiales, han crecido en todos los distritos excepto Santa Fe y Córdoba y se ha mantenido en Chaco y La Pampa. La consulta en establecimientos oficiales es mayor en provincias patagónicas o centrales y es menor en las provincias del norte como Santiago del Estero, Formosa, Chaco. Parece que continúa cumpliéndose la ley de Hart la cual sostiene que "el acceso a atención médica de calidad disminuye en proporción inversa a su necesidad en la población, y ello se cumple más intensamente donde las fuerzas del mercado son más fuertes". (20). 


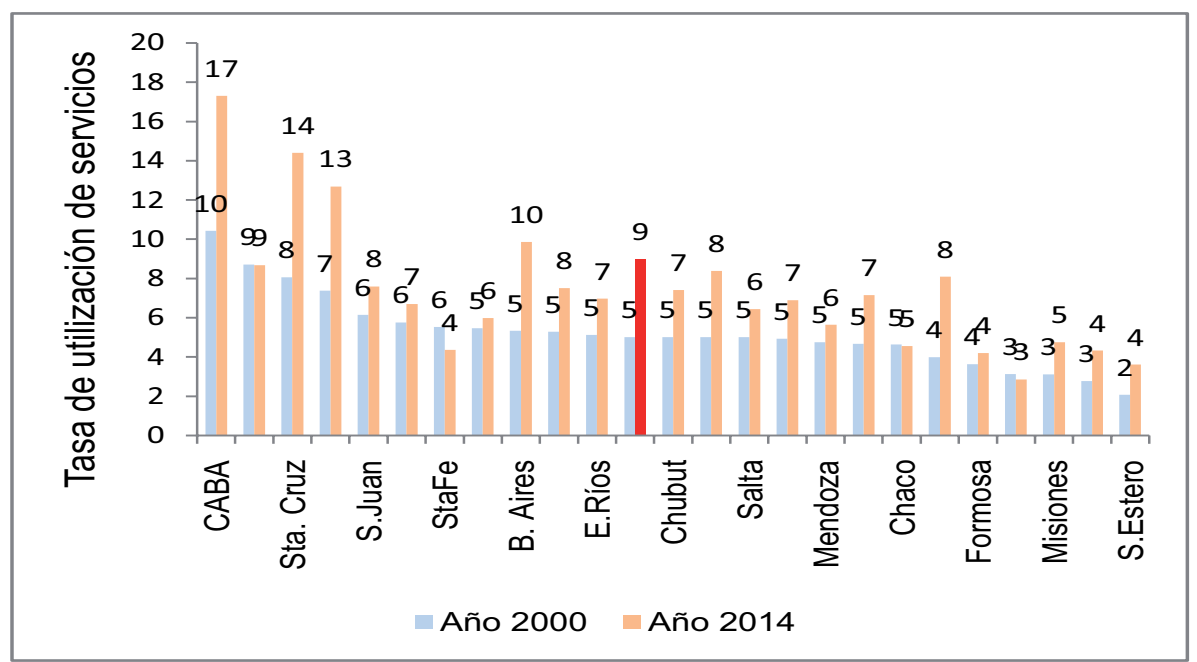

Gráfico $\mathrm{N}^{\circ}$ 20: Tasa de uso de consulta ambulatoria en servicios oficiales. Argentina 2000 у 2013

Elaboración propia. Fuentes de datos: Ministerio de Salud. Sistema Estadístico de salud. Año 2016. (21).

El gráfico $\mathrm{N}^{\mathrm{o}} 21$ apoya este enunciado pues la gente acude a los establecimientos oficiales en los lugares donde hay más cobertura de obra social (el gráfico mide como la falta de cobertura crece junto a la retracción de las tasas de uso en los establecimientos públicos destinados precisamente a atender a esa franja poblacional). El cuadro podría llevar a preguntarnos si en las provincias donde hay mayor empleo formal, se prestan mejores servicios estatales. Esa relación entre utilización y calidad sin embargo no se analiza en este trabajo.

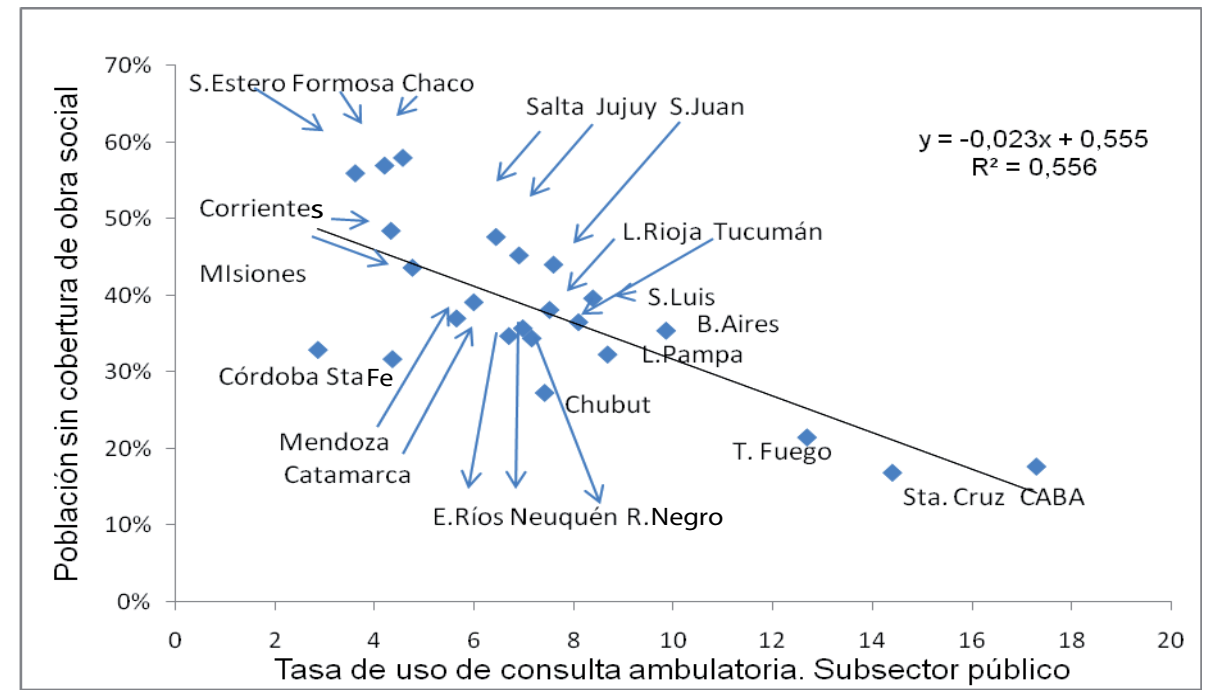

Gráfico $\mathrm{N}^{\mathrm{0}}$ 21: Relación entre la tasa de uso de consulta ambulatoria en establecimientos oficiales y ausencia de cobertura de obra social

Elaboración propia. Fuentes de datos: Ministerio de Salud. Argentina OPS 2015 (6).

Sistema Estadístico de salud. Año 2016. (21).

Como antes se indaga finalmente en la relación entre la utilización de los servicios y la expectativa de vida. El coeficiente es del 28,6 \% casi similar al de la razón de médicos 
por habitantes. Por otro lado el R2 entre utilización de servicios y mortalidad infantil es de $19,4 \%$.

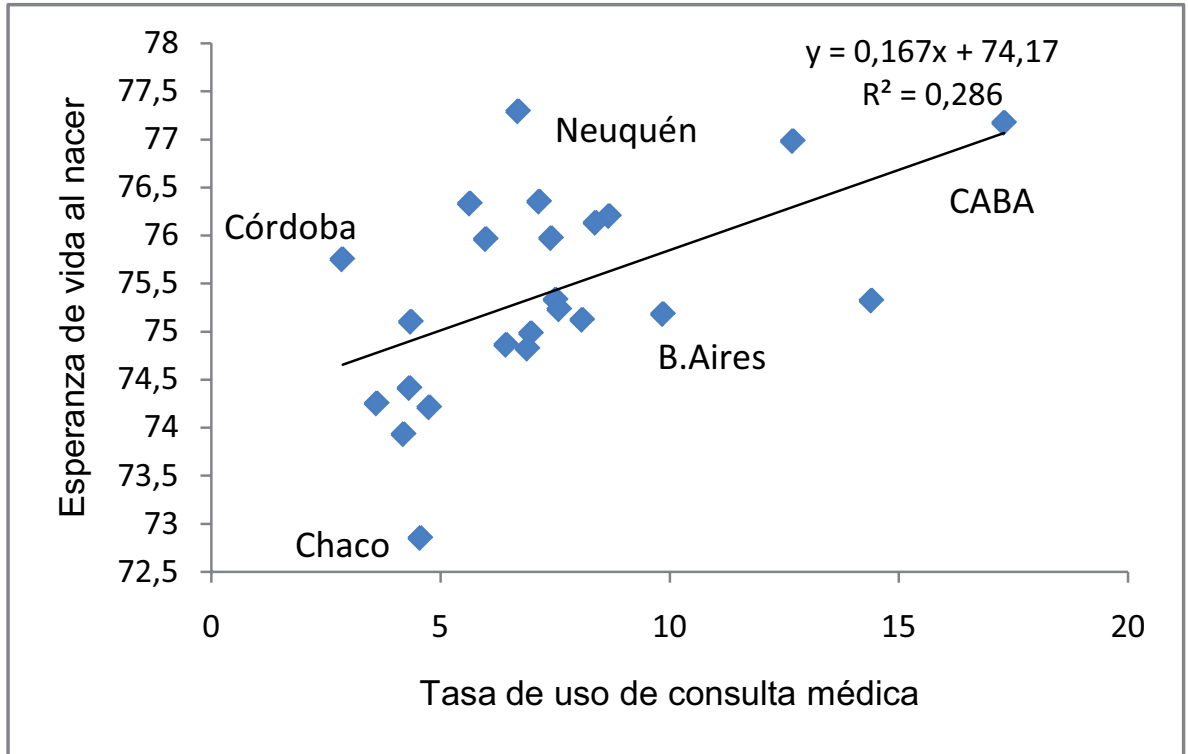

Gráfico $\mathrm{N}^{\mathrm{o}}$ 22: Relación entre la esperanza de vida al nacer y la tasa de uso de consulta ambulatoria en establecimientos oficiales

Elaboración propia. Fuentes de datos: Ministerio de Salud. Argentina OPS 2015 (6).

Sistema Estadístico de salud. Año 2016. (21).

Para dar una visión mas integrada de los análisis bivariados anteriores se presenta finalmente el grafico de una exploración factorial incluyendo además de las variables utilizadas la provisión de cloacas, agua y establecimientos públicos con y sin internación para el año 2000

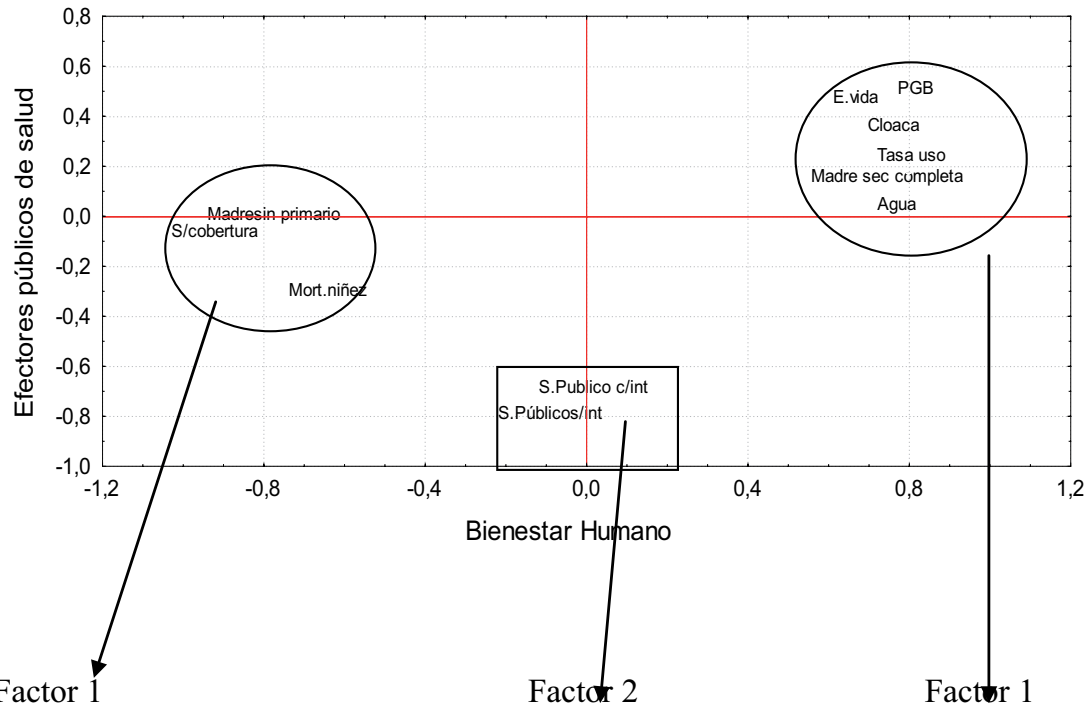

Gráfico $\mathrm{N}^{\mathrm{o}}$ 23: Relación bidimensional entre variables sociodemográficas y servicios de salud. Argentina 2000

Elaboración propia. Fuentes de datos Ministerio de Salud de la Nación: Argentina Indicadores básicos de salud Argentina OPS 2003(6). Estadísticas básicas 2001. (11). 
Se observa en el gráfico $\mathrm{N}^{\mathrm{o}} 23$ dos ejes que dan una imagen sintética de la distribución de las variables. En el eje 1 que capta más del 53\% de las variaciones asociadas se distinguen dos semiejes, a la derecha aparecen juntas: alta esperanza de vida al nacer, madre con secundario completo, PGB alto, alta tasa de uso de consulta ambulatoria en servicios públicos y presencia de agua potable y cloaca. En el semieje negativo es decir a la izquierda del eje horizontal, las correlaciones negativas a esas variables son: madre con primario incompleto, ausencia de cobertura de obra social, muerte de la niñez. En el segundo eje (el vertical), aparecen servicios públicos con y sin internación es decir sin vinculación a las variables anteriores. La lectura del gráfico indicaría que la mortalidad de la niñez no tiene que ver con los servicios de salud sino con la educación de la madre, la ausencia de trabajo estable y de servicios de saneamiento. Esto avala las palabras de McKeown, (en Deaton, p.112) (2), quien concluyó que la medicina no era muy útil y que las raíces de la mejoría en la salud consistían en el progreso social y económico. Siendo la pobreza y las privaciones las causas fundamentales de la salud deficiente. Para nosotros la variable clave parece ser la educación de la mujer.

El gráfico $\mathrm{N}^{\circ} 24$ refiere al año 2014, presenta en el primer eje el mismo cuadro de una década y media antes, a la derecha las madres con secundario completo, agua potable, cloacas, PGB alto y altas tasas de uso de consulta ambulatoria en servicios públicos. A la izquierda del eje la mortalidad de la niñez acompañada de madres analfabetas o con primario incompleto y ausencia de cobertura de obras sociales. En el segundo eje se destaca sólo el semieje positivo y asocia el alto número de médicos y camas. La diferencia con el gráfico anterior entonces, es que el segundo eje le da más relevancia a los médicos y las camas que a los efectores públicos de salud. Porque el sector privado gana protagonismo en estos quince años ha sido el motivo de un libro reciente de los autores. (22)

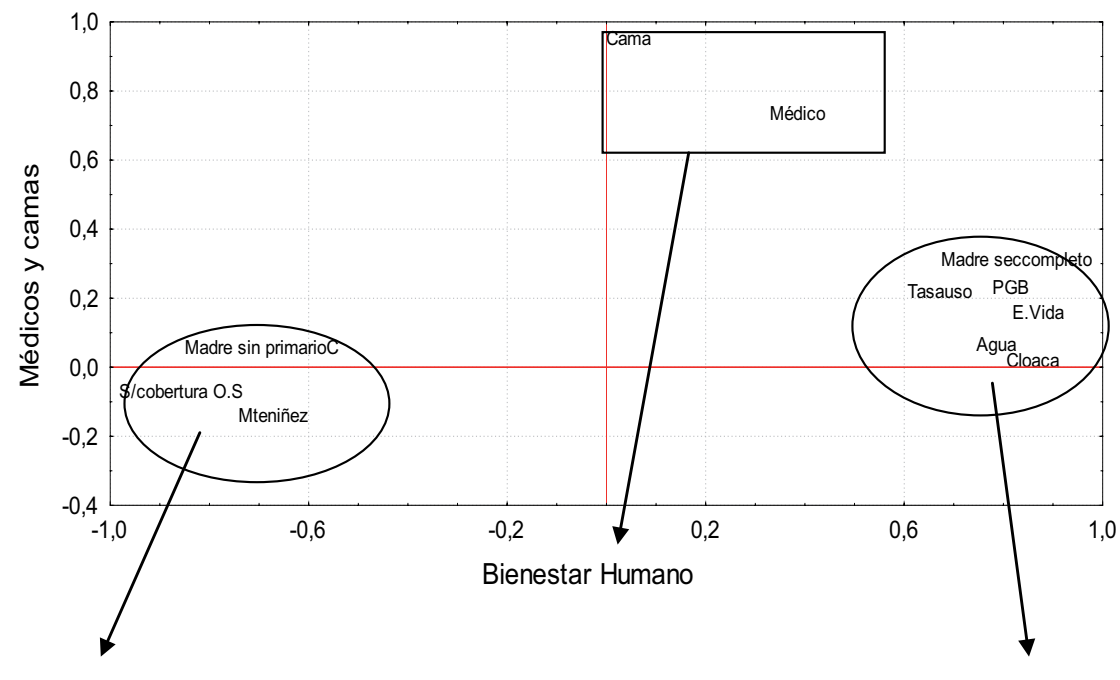

Primer eje

Segundo eje

Primer eje

Gráfico No 24: Relación bidimensional de variables sociodemográficas y servicios de salud. Argentina 2014 


\section{Conclusiones}

El estudio consta de dos partes. En la primera se hizo una descripción de los cambios ocurridos en Argentina en la expectativa de vida durante la primera década del siglo y de la mortalidad de la niñez durante el período 2000-2014. Luego se indagó en la asociación de la expectativa de vida y la mortalidad de la niñez con el producto geográfico bruto por habitante, la educación de la mujer, la razón de médicos por habitantes y la tasa de utilización de la consulta en establecimientos públicos.

La conclusión central es que la salud pública medida por la expectativa de vida al nacer ha hecho un enorme progreso y que ese progreso se reparte con valores muy altos entre Provincias de muy desigual ingreso por habitante. La esperanza de vida al nacer en la presente década es de 75 años y la diferencia entre las dos provincias extremas de cuatro años. Resulta igualmente significativo que la desigualdad entre Provincias ha disminuido y que junto a una mayor expectativa de vida está ocurriendo el envejecimiento de la muerte medida por la participación de dos grupos etarios (menores de 5 años y mayores de 60). Entre 2000 y 2014, la tasa de mortalidad de menores de cinco años bajó del 19,2 \%o nacidos vivos al 12,2 \%o o sea una caída del 37\%. Los menores de 5 años mueren principalmente por causas perinatales (44\%) y la segunda causa de muerte son las enfermedades congénitas (26\%). Argentina se halla entre los países donde la muerte de mayores de 60 años es más importante numéricamente que la muerte de menores de 5 años. La estructura de la mortalidad por tumores en mayores de 60 años permanece casi inalterada.

La asociación de la expectativa de vida al nacer y la mortalidad infantil con el producto bruto geográfico muestra que este determina casi un $50 \%$ de la primera variable pero apenas un $35 \%$ de la mortalidad en la niñez. Por otro lado, no explica porque provincias con los mismos progresos en años de expectativa de vida ganados se encuentran en valores muy diferentes en relación al producto bruto geográfico por habitante. Se buscó indagar entonces la asociación con la educación de la mujer.

Durante los catorce años en estudio disminuyó el porcentaje de mujeres sin instrucción, con primario incompleto o completo y aumento el porcentaje de mujeres con secundario y terciario/ universitario completo. El coeficiente de determinación entre mujeres con secundario completo y expectativa de vida fue del $60 \%$, pero solo del $33 \%$ en relación a la mortalidad de la niñez. Esto, como en el caso del producto geográfico bruto tal vez se deba a la participación de la mortalidad por causas congénitas.

Finalmente la asociación entre la razón de médicos por habitantes y la tasa de utilización de consulta en establecimientos oficiales con la expectativa de vida dio una muy débil asociación inferior al $30 \%$. Por otro lado la relación entre razón de médicos y mortalidad de la niñez fue del $4 \%$ y no significativa, mientras que fue del $19 \%$ con la tasa de consulta médica. Un análisis multivariante revela que la mortalidad de la niñez asocia en forma positiva con la ausencia de cobertura de obra social, madres analfabetas o con primaria incompleta y en forma negativa con el PGB por cápita, madres con secundario completo, alta tasa de uso de consulta en servicios públicos y servicios de saneamiento, mientras que en un segundo eje se ubican los médicos y las camas. La diferencia entre el año 2000 y el 2014 es que los médicos y las camas vienen a sustituir los establecimientos oficiales con y sin internación lo que sugeriría un avance de la oferta privada de servicios sobre la pública. Lo expuesto señala que la variable más importante en estos avances en salud es la educación de la madre que completó el secundario.

La salud pública por un lado, debe consolidar los indicadores logrados y por el otro encarar el núcleo duro de la mortalidad de la niñez donde predominan las malformaciones congénitas y las perinatales. Este desafío es doble, por un lado porque la atención es cada vez más compleja y costosa, y por el otro porque los pacientes con secuelas pasan a ser frecuentes. Esto significa que la salud pública debe posicionarse frente a un futuro que 
demandará más complejidad, especialistas y recursos. Se abren así posibles estrategias diferentes. Por un lado consolidar la oferta de servicios dentro de los prestadores públicos y por el otro armar alianzas estratégicas con el sector privado.

\section{Bibliografía}

1. Cluster Salud. OMS. Aumenta esperanza de vida pero persiste desigualdad en salud. Cluster Salud. 2016, Jun, 21. (En línea). Disponible http//www.clustersalud. americaeconomia.com.

2. Deaton A. El Gran Escape: salud, riqueza y los orígenes de la desigualdad. Buenos Aires: Fondo de Cultura Económica; 2015.

3. Organización de Naciones Unidas. Programa de las Naciones Unidas para el Desarrollo. La verdadera riqueza de las Naciones: Caminos al desarrollo humano. Nueva York: PNUD; 2010. (En línea). Disponible http//www.undp.org.ar/docs/ prensa. Acceso 20 de abril de 2016.

4. Mason Paul. Postcapitalismo Hacia un Nuevo Futuro. Paidós. Buenos Aires, 2016: p16

5. Banco Mundial. Washington D.C.: W.B; 2011. (En línea). Disponible en http//www. worldbank.org/data. Acceso 20 de abril de 2016.

6. República Argentina. Ministerio de Salud, Organización Panamericana de la Salud. Datos y estadísticas. Indicadores básicos de salud de Argentina. Buenos Aires: OPS Argentina; 2003 y 2015. (En línea). Disponible http//www.deis. gov.ar

7. Fondo de las Naciones Unidas para la infancia. Estadísticas. Nueva York; UNICEF; 2015. (En línea). Disponible en http// www.unicef.org. Acceso 20 de abril de 2015.

8. Organización de Naciones Unidas. Programa de las Naciones Unidas para el Desarrollo. Trabajo al servicio del desarrollo humano. Nueva York: PNUD; 2015. (En línea). Disponible http//www.undp.org.ar/docs/prensa. Acceso 20 de abril de 2016.

9. Organización de Naciones Unidas. Departamento de Asuntos Económicos y Sociales de la Secretaría de las Naciones Unidas. Objetivos de Desarrollo del Milenio. Informe de 2015; Jun. MDG. (En línea). Disponible en http://un.org/es/ mdg/. Acceso 20 de abril de 2015.

10. Organización Mundial de la Salud. Estadísticas Sanitarias Mundiales. Washington D.C.: OMS; 2015. (En línea). Disponible en http: //www.who.int/whosis/gho/ publications/Word_health.../2015.

11. República Argentina. Ministerio de Salud. Secretaria de Políticas y Regulación e Institutos. Dirección de Estadísticas e Información de Salud. Estadísticas vitales e información básica; 2001, 2002, 2003, 2004, 2005, 2006, 2007, 2008, 2009, 2010, 2011, 2012, 2013, 2014, 2015. (En línea). Disponible http/. www.deis.gov.ar.

12. Ministerio de Hacienda y Finanzas Públicas de la Nación. Dirección Nacional de Relaciones Económicas con las Provincias. Sistema de Indicadores de Desarrollo Provincial (SIDEP). Disponible http//www.indec. gov.ar. Acceso 20 de abril de 2016. República Argentina.

13. Ministerio de Hacienda y Finanzas Públicas de la Nación. Encuesta permanente de hogares, Primer trimestre 2015. Disponible http//www.indec. gov.ar. Acceso $20 \mathrm{de}$ abril de 2016. República Argentina.

14. United Nations. Indicators for monitoring the Millenium Development Goals. Definitions, rationale, concepts and sources. New York: United Nations, 2003.

15. Ministerio de Hacienda y Finanzas Públicas de la Nación. Oferta y demanda global. Disponible http//www.indec.gov.ar. Acceso 20 de abril de 2016. República 
Argentina.

16. Secretaría Distrital de Salud de Bogotá. Saludcapital.gov.co1999

17. Nguyen Ngoc N, Merialdi M,Aleem H,Carroli G, Purwar M, Zavaleta $\mathrm{N}$, Campodónico $\mathrm{L}$ et al. Causas de mortinatalidad y de mortalidad neonatal precoz: datos de 7993 embarazos en seis países en desarrollo. Boletín de la Organización Mundial de la Salud OMS, 2016.

18. Paris E (2011) Gran estudio sobre la muerte perinatal en el mundo bebesymas.com.

19. República Argentina. Ministerio de Salud. Observatorio Federal de Recursos Humanos en Salud Los recursos humanos de salud en Argentina. (En línea). Disponible http/. www.msal.gov.ar/observatorio

20. Hart J.T. (1971). The inverse care law. Lancet; I: 405-412.

21. República Argentina. Ministerio de Salud. Sistema estadístico de Salud. Consultas ambulatorias en establecimientos oficiales. Argentina 2013. Mar2016 (En línea). Disponible http/. www.deis.gov.ar

22. Tafani R, Gaspio N. Medicina Privada y Salud Pública: La Lógica del Capital y el Deterioro Público en la Atención Médica. Editorial Brujas 2015. Córdoba Argentina. 